Mon. Not. R. Astron. Soc. 000, 1 20 (2010) Printed 26 May $2018 \quad$ (MN LATEX style file v2.2)

\title{
Tidal Truncation of Circumplanetary Discs
}

\author{
Rebecca G. Martin and Stephen H. Lubow ${ }^{\star}$ \\ Space Telescope Science Institute, 3700 San Martin Drive, Baltimore, MD 21218, USA
}

26 May 2018

\begin{abstract}
We analyse some properties of circumplanetary discs. Flow through such discs may provide most of the mass to gas giant planets, and such discs are likely sites for the formation of regular satellites. We model these discs as accretion discs subject to the tidal forces of the central star. The tidal torques from the star remove the disc angular momentum near the disc outer edge and permit the accreting disc gas to lose angular momentum at the rate appropriate for steady accretion. Circumplanetary discs are truncated near the radius where periodic ballistic orbits cross, where tidal forces on the disc are strong. This radius occurs at approximately $0.4 r_{\mathrm{H}}$ for the planet Hill radius $r_{\mathrm{H}}$. During the T Tauri stage of disc accretion, the disc is fairly thick with aspect ratio $H / r \gtrsim 0.2$ and the disc edge tapering occurs over a radial scale $\sim H \sim 0.1 r_{\mathrm{H}}$. The disc fluid equations can be rescaled in the Hill approximation to a form similar to the flow equations for a disc in a binary star system with a mass ratio of unity. For a circular or slightly eccentric orbit planet, no significant resonances lie within the main body of the disc. Tidally driven waves involving resonances nonetheless play an important role in truncating the disc, especially when it is fairly thick. We model the disc structure using one dimensional time-dependent and steady-state models and also two dimensional SPH simulations. The circumplanetary disc structure depends on the variation of the disc turbulent viscosity with radius and is insensitive to the angular distribution of the accreting gas. Dead zones may occur within the circumplanetary disc and result in density structures. If the disc is turbulent throughout, the predicted disc structure near the location of the regular Jovian and Saturnian satellites is smooth with no obvious feature that would favor formation at their current locations. It may be possible that substructure, such as due to variations in the disc turbulence, could lead to the trapping of migrating satellites.
\end{abstract}

Key words: accretion, accretion discs - planets and satellites: formation - planetary systems - planet-disk interactions

\section{INTRODUCTION}

In the core accretion model of planet formation, at the earliest stages of giant planet formation, the planet is fully embedded in the gaseous disc that orbits the central star (Mizuno 1980; Pollack et al. 1996; Hubickyi, Bodenheimer \& Lissauer 2005; Papaloizou \& Nelson 2005). At later stages, during run-away-gas accretion, tidal forces due to the planet open a gap in the disc, typically when the planet reaches a mass of order Neptune's mass (Lin \& Papaloizou 1986; Bate et al. 2003; D'Angelo, Henning \& Klev 2002). The gap opening does not necessarily imply that the gas flow on to the planet has ceased (Artvmowicz \& Lubow 1996). The gap structure is determined by the properties of dynamical flow within it. The relatively low density of gas in the gap results from the relatively high flow radial velocities towards the star as a consequence of the disturbance caused by the planet. Outside the gap region, the gas radial drift occurs on the much slower viscous timescale, resulting in much higher densities than occur in the gap. Some studies suggest that most of the inflowing gas just outside

\footnotetext{
* E-mail: rmartin@stsci.edu; lubow@stsci.edu
} 
the gap is accreted by a planet whose mass is comparable to or less than Jupiter's for typical disc parameters (Bryden et al. 1999; Kley 1999; Lubow, Seibert \& Artymowicz 1999; Lubow \& D'Angelo 2006).

During this gap phase, the planetary radius is typically much smaller than its Hill (tidal) radius. Circumplanetary discs may form as gas flowing through the gap on to a planet carries some angular momentum about the planet (Lubow et al. 1999; Bate et al. 2003; D'Angelo et al. 2002). As a result, the inflowing gas has too much angular momentum to directly strike the planet and instead forms a disc about it. Most of the mass of Jupiter may have been acquired in the gap stage through gas flow that involves a circumplanetary disc.

There are at least two motivations for studying circumplanetary discs. The observational detection of a disc orbiting an extra-solar gas giant planet would provide important evidence about the process of planet formation. The determination of their expected properties provides constraints for their detection. A second motivation is to better understand satellite formation, since circumplanetary discs are likely sites of satellite formation. Satellites that orbit Jupiter and Saturn are classified into two groups. The regular satellites have low eccentricity and low orbital inclination to the equatorial plane of their planet. They are thought to have formed in the circumplanetary disc (Lunine \& Stevenson 1982; Canup \& Ward 2002; Mosqueira \& Estrada 2003). The second type, the irregular satellites, can have a high eccentricity and high orbital inclinations. They can orbit either progradely or retrogradely with respect to the planet spin (Grav et al. 2003). The circumplanetary disc may capture these satellites though gas drag (Pollack, Burns \& Tauber 1979; Ćuk \& Burns 2004), although the disc does not play such a role in the Nice model (e.g. Gomes et al. 2005). The circumplanetary accretion disc plays a vital role in satellite formation. The regular satellite systems of Jupiter and Saturn extend over a small fraction of their respective Hill radii, to less than $0.06 r_{\mathrm{H}}$. This fact has motivated a dynamical explanation in terms of circumplanetary disc sizes (e.g. Canup \& Ward 2002; Mosqueira \& Estrada 2003). We discuss this in more detail in Section 8.

Previous studies have considered the role of the angular momentum (about the planet) of the inflowing gas in determining the disc structure, in particular the disc outer radius. This picture has some intuitive appeal, since no disc would form, i.e., the disc radius would be zero, if the inflowing gas has zero angular momentum. Quillen \& Trilling (1998) suggested that after the planet has opened a gap, the circumplanetary disc extends to $r \approx r_{\mathrm{H}} / 3$. This result is based on the initial angular momentum of gas whose flow relative to the planet is slow near the planet's Hill sphere and subsequently accelerates inward towards the planet while conserving its angular momentum about the planet. Canup \& Ward (2002) and Ward \& Canup (2010) considered cases where the angular momentum of the inflowing gas is considerably smaller.

Lissauer (1995) (see also Lissauer et al. 2009) suggested that the circumplanetary disc structure prior to or during gap opening could account for the location of regular satellites. This model was also based on the concept that the disc structure is determined by the angular momentum of infalling gas about the planet, with an implied smaller value of disc radius of $r=r_{\mathrm{H}} / 48$. This paper concentrates on the former case, the case of a circumplanetary disc in the presence of gap opening. The results of this paper may also apply to this case. We briefly discuss this point again below equation (4) and in Section 8

This radius characteristic of the angular momentum of the accreting gas is useful for providing an estimate of the conditions required for circumplanetary disc formation. For a disc to form about a planet with a gap, the planetary radius must be smaller than this value. Violating this constraint for disc formation would require very short orbital periods, of order $1.5 \mathrm{~d}$, for a planet of Jupiter's radius.

However, it is not clear that this radius characteristic of the angular momentum of the accreting gas is important for determining the disc radius or even has much influence of the disc structure. The reason has to do with the requirements of angular momentum loss from a steady-state disc. The process of angular momentum transport in circumplanetary discs has up to now been largely ignored. The work by Canup \& Ward (2002) and Ward \& Canup (2010) did consider the angular momentum issue, as will be discussed further in Section 6.5. Mosqueira \& Estrada (2003) briefly discuss the possible role of resonances in truncating the disc. For the disc to accrete in a steady-state manner, gas within it must continuously lose angular momentum. These studies recognized that the disc would extend beyond the radius in which mass is injected in order to account for the requirements of angular momentum conservation. The angular momentum issue has been previously encountered in the case of discs in mass exchange binary star systems (e.g. Papaloizou \& Pringle 1977). The inflowing gas from the mass losing star is captured within the Roche lobe of the companion star. This gas has some angular momentum that often results in the formation of disc about the companion. The picture that has resulted is that the disc spreads outward by viscous torques until it reaches a radius at which tidal torques from the companion are sufficient to remove the angular momentum at the rate required for steady state accretion. The disc structure is insensitive to the angular momentum of the accreting gas. The case of a planet accreting gas can be considered to be a binary of extreme mass ratio. The accretion disc model in the binary star case suggests a somewhat different picture of how the accretion process operates than has been previously considered for circumplanetary discs. We consider the accretion disc model of circumplanetary discs in this paper.

Some recent three-dimensional simulations have analysed the flow in circumplanetary discs. For example, Avliffe \& Bate (2009) simulated gas accretion by protoplanets in three-dimensions with gas self-gravity and radiation transfer to investigate the properties of circumplanetary discs. They determined the disc scale height to be $H / r>0.2$ and found the disc size to be about $0.35 r_{\mathrm{H}}$. They attributed this value of the disc radius as following from the estimate of Quillen \& Trilling (1998), for which the radius is determined by the angular momentum of the accreting gas. 
Machida (2009) also performed three-dimensional calculations of circumplanetary discs. For a Jupiter mass planet, he found a peak in the surface density at about 21 protoplanetary radii (or $0.028 r_{\mathrm{H}}$ ) from the protoplanet centre. According to these results, the peak is due to the balance between the centrifugal force and the gravity of the protoplanet. Such small scale features might then correspond to the formation sites of the regular satellites around Jupiter and Saturn. However, this peak in the surface density was not reproduced by Ayliffe \& Bate (2009) who found that the surface density decreases monotonically with radius from the protoplanet.

We analyse the general properties of circumplanetary discs by the use of some simplified models, in order to understand the physical processes that affect disc structure. In Section 2, we estimate the scale height and temperature of the disc. In Section 3 we consider a ballistic disc model consisting of periodic particle orbits around a planet. In Section 4 we examine the circumplanetary fluid disc evolution equations in the Hill approximation and discuss their relationship to the binary star case. In Section 5 we consider whether resonances could lie within a circumplanetary disc. In Section 6 , we analyse the effects of a tidal torque on a purely viscous disc. In Section $\square$ we describe results of a model that includes the effects of gas pressure by means of some SPH simulations. Section 8 contains a discussion and the conclusions.

\section{CIRCUMPLANETARY DISC PROPERTIES}

We consider a system with a planet of mass $M_{\mathrm{p}}$ in a circular orbit around a star of mass $M_{\mathrm{s}}$ at a separation $a$. The Hill sphere is the approximate region where the planet's gravity dominates that of the star and its radius is given by

$$
r_{\mathrm{H}}=a\left(\frac{\mu}{3}\right)^{\frac{1}{3}}
$$

where $\mu=M_{\mathrm{p}} /\left(M_{\mathrm{p}}+M_{\mathrm{S}}\right) \ll 1$. The sound speed in an ideal gas is

$$
c_{\mathrm{s}}=\sqrt{\frac{k T}{\mu_{\mathrm{m}} m_{\mathrm{H}}}} \approx 10^{6} \sqrt{\frac{T}{10^{4}}} \mathrm{~cm} \mathrm{~s}^{-1},
$$

where $k$ is the Boltzmann constant, $T$ is the temperature, $\mu_{\mathrm{m}}$ is the mean molecular weight and $m_{\mathrm{H}}$ is the mass of a hydrogen atom. The surface temperature of a steady state accretion disc around an object of mass $M$ at a radius $r$ is given by

$$
\sigma T^{4}=\frac{3}{8 \pi} \frac{G M \dot{M}}{r^{3}}
$$

(Pringle 1981) where $\sigma$ is the Stefan-Boltzmann constant.

We estimate the temperature of the accreting gas with the disc. Similar approaches were taken by Canup \& Ward (2002). Consider a disc orbiting a solar mass star that is accreting gas at a rate of $10^{-8} \mathrm{M}_{\odot} \mathrm{yr}^{-1}$, a typical value inferred from observations of T Tauri stars. Equation 3 implies that at a distance from the central star of 5 AU, the disc has a temperature of about $26 \mathrm{~K}$, ignoring effects of stellar heating. This temperature corresponds to a disc aspect ratio $H / r \simeq 0.04$.

The aspect ratio of the circumplanetary disc taken with respect to the planet is

$$
\left(\frac{H}{r}\right)_{\mathrm{p}}=\frac{c_{\mathrm{s}}}{\Omega r}=0.3\left(\frac{r}{r_{\mathrm{H}} / 3}\right)^{\frac{1}{8}}\left(\frac{M_{s}}{M_{\odot}}\right)^{\frac{1}{24}}\left(\frac{M_{\mathrm{p}}}{M_{\mathrm{J}}}\right)^{-\frac{1}{3}}\left(\frac{a}{5 \mathrm{AU}}\right)^{\frac{1}{8}}\left(\frac{\dot{M}_{\mathrm{p}}}{10^{-8} \mathrm{M}_{\odot} \mathrm{yr}^{-1}}\right)^{\frac{1}{8}},
$$

where $M_{\mathrm{J}}$ is the mass of Jupiter and $r$ is the distance from the planet. (There is a small correction due to the vertical gravity of the star that we ignore.) This aspect ratio is much larger than the value for the circumstellar disc, $(H / r)_{\mathrm{s}} \simeq 0.04$. We have taken the disc sound speed $c_{\mathrm{s}}$ to be given by its value at the disc surface and have ignored any increase at the midplane with optical depth. The relatively high value of $(H / r)_{\mathrm{p}}$ is due to the higher gas temperatures and the typically smaller $\Omega r$ values in the circumplanetary disc case. The value of the disc aspect ratio from equation (4) is similar to the values obtained in the simulations by Ayliffe \& Bate (2009). They found no evidence for circumplanetary discs in their simulations of low mass planets (less than 100 Earth masses). They attributed this result to the weakness of the planet's vertical gravity compared to pressure, as can be seen in equation (4). For a sufficiently low mass planet, $(H / r)_{p}$ can be large enough that the concept of a disc breaks down and the gas around the planet is essentially spherical. As is well known, the circumplanetary disc temperatures at these accretion rates are too high to explain the existence of the icy satellites of Jupiter and Saturn (Canup \& Ward 2002). However, in a slower accretion phase as proposed by Canup \& Ward (2002) and further investigated by Barr \& Canup (2008), the temperature would be lower and $H / r$ would be smaller, $H / r \sim 0.1$. We discuss this further in Section 8

We compare the surface density in the circumplanetary disc, $\Sigma_{\mathrm{p}}$, to the surface density in the circumstellar disc, $\Sigma_{\mathrm{s}}$. We assume that most of the mass being accreted through the circumstellar disc outside the orbit of the planet is accreted by it. As discussed in the Introduction, this efficient gas capture has been found in simulations of Jupiter mass planets that orbit solar mass stars. The mass transfer rate through a steady state disc is given by $\dot{M} \propto \nu \Sigma($ Pringle 1981), where the viscosity is parametrised with the $\alpha$ prescription so that

$$
\nu=\alpha c_{\mathrm{s}} H=\alpha\left(\frac{H}{r}\right)^{2} r^{2} \Omega
$$


(Shakura \& Sunyaev 1973) where $\Omega=\sqrt{G M_{\mathrm{p}} / r}$. It then follows that

$$
\frac{\Sigma_{\mathrm{s}}}{\Sigma_{\mathrm{p}}} \sim \frac{\nu_{\mathrm{p}}}{\nu_{\mathrm{s}}} \sim \mu^{2 / 3} \frac{\alpha_{\mathrm{p}}}{\alpha_{\mathrm{s}}} \frac{\left(\frac{H}{r}\right)_{\mathrm{p}}^{2}}{\left(\frac{H}{r}\right)_{\mathrm{s}}^{2}} \sqrt{\frac{r}{r_{\mathrm{H}}}}
$$

where $\nu_{\mathrm{s}}$ and $\nu_{\mathrm{p}}$ are the viscosities in the circumstellar and circumplanetary discs respectively, and $\alpha_{\mathrm{s}}$ and $\alpha_{\mathrm{p}}$ similarly refer the $\alpha$ values in the discs.

Consider a solar mass star and a Jupiter mass planet, $\mu=10^{-3}$, and circumstellar and circumplanetary disc aspect ratios of 0.04 and 0.3 respectively, as discussed above. For $r=0.2 r_{\mathrm{H}}$ and equal $\alpha$ values $\left(\alpha_{\mathrm{s}}=\alpha_{\mathrm{p}}\right)$ in the above equation, the ratio of the surface densities is $\Sigma_{\mathrm{s}} / \Sigma_{\mathrm{p}} \sim 0.25$. So the circumplanetary disc surface density is somewhat higher that the local circumstellar disc density. Circumstellar discs could under some circumstances contain dead zones, regions where the disc ionization is too low for the magneto-rotational instability to operate as a source of turbulence (Gammie 1996). Dead zones operate where the temperature is sufficiently low that thermal ionization is weak (less than about $10^{3}$ degrees) and where the surface densities are high enough that external sources of ionization such as cosmic rays do not penetrate far below the disc surface. For expected conditions in a circumstellar disc, dead zones may extend from a few tenths of an AU to several AU, in some cases beyond Jupiter's orbital radius (e.g. Terquem 2008). The model here suggests that the conditions for dead zone formation within a circumplanetary disc are perhaps more favorable than in the nearby circumstellar disc gas. The reason is that the circumstellar disc surface densities are higher than in the nearby circumstellar disc gas, while the temperatures are low enough to avoid sufficient ionisation in much of the circumplanetary disc. A difference in the properties of turbulence in the two discs violates our assumption of $\alpha_{\mathrm{s}}=\alpha_{\mathrm{p}}$. But, it does so in a way that may further enforce the importance of dead zones in the circumplanetary disc. That is, $\alpha_{\mathrm{s}} / \alpha_{\mathrm{p}}$ would be expected to increase, leading to a decrease in $\Sigma_{\mathrm{s}} / \Sigma_{\mathrm{p}}$. We do not pursue the possibility of dead zones further in our disc models described later and assume a simple viscous disc.

The viscous timescale in the disc is

$$
t_{\nu_{\mathrm{p}}} \sim \frac{r^{2}}{\nu_{\mathrm{p}}} \sim \frac{1}{\alpha(H / r)_{\mathrm{p}}^{2} \Omega} \sim 10^{3} \mathrm{P}\left(\frac{10^{-3}}{\alpha}\right)\left(\frac{0.3}{H / r}\right)^{2}\left(\frac{r}{r_{\mathrm{H}}}\right)^{3 / 2},
$$

where $P$ is the orbital period of the planet. So we expect that such discs should be viscously relaxed for Jupiter and Saturn. This result has the implication that the viscous disc flow can be regarded to be in a steady state.

A crude estimate of the steady state luminosity ratio of the circumplanetary to circumstellar discs in a system with a Jupiter mass planet is

$$
\frac{L_{\mathrm{p}}}{L_{\mathrm{s}}} \sim \frac{\dot{M}_{\mathrm{p}} M_{\mathrm{p}} R_{\mathrm{s}}}{\dot{M}_{\mathrm{s}} M_{\mathrm{s}} R_{\mathrm{p}}}
$$

where $R_{\mathrm{p}}$ and $R_{\mathrm{s}}$ are the planet and star radii, respectively. For $\dot{M}_{\mathrm{p}} \sim 1-10 \dot{M}_{\mathrm{s}}, M_{\mathrm{p}} \sim 0.001 M_{\mathrm{s}}$, and $R_{\mathrm{p}} \sim 0.1 R_{\mathrm{s}}$, we estimate $L_{\mathrm{p}} / L_{\mathrm{s}}$ to be of order a few percent. We see that circumplanetary discs are not very bright. We discuss this further in Section 8

\section{BALLISTIC PARTICLE PERIODIC ORBITS AROUND THE PLANET}

In this section we consider ballistic particle orbits in a planet-star system in order to find their nonKeplerian angular velocity and where the orbits begin to cross.

\subsection{Ballistic Equations}

As a simple two-dimensional disc model, we consider ballistic particles orbiting a planet of mass $M_{\mathrm{p}}$ in the corotating frame of the star-planet system with a star of mass $M_{\mathrm{s}}$. The particles lie in the star-planet orbit plane. For such a model to represent a low pressure (cold) steady state fluid disc, each orbit must be periodic in the corotating frame and be nonintersecting, either with itself or neighboring orbits, in order that the velocity be single-valued in space. In addition, the orbit must be stable. The equation of motion of a ballistic particle at position $\boldsymbol{r}$ for potential $\phi$ in the corotating frame is

$$
\ddot{\boldsymbol{r}}+2 \boldsymbol{\Omega}_{\mathrm{p}} \times \dot{\boldsymbol{r}}=-\nabla \phi,
$$

where $\boldsymbol{\Omega}_{\mathrm{p}}$ is the angular velocity of the planet. The potential due to the rotation of the frame and the gravity of the planet and the star is given by

$$
\phi=-\frac{\left|\boldsymbol{r}-\boldsymbol{r}_{\mathrm{cm}}\right|^{2} \Omega_{\mathrm{p}}^{2}}{2}-\frac{G M_{\mathrm{p}}}{\left|\boldsymbol{r}-\boldsymbol{r}_{\mathrm{p}}\right|}-\frac{G M_{\mathrm{s}}}{\left|\boldsymbol{r}-\boldsymbol{r}_{\mathrm{s}}\right|},
$$

where $\boldsymbol{r}_{\mathrm{cm}}, \boldsymbol{r}_{\mathrm{p}}$ and $\boldsymbol{r}_{\mathrm{s}}$ are the position vectors of center of mass, the planet, and the star respectively. The angular velocity of the star-planet binary system is

$$
\Omega_{\mathrm{p}}=\left(\frac{G\left(M_{\mathrm{s}}+M_{\mathrm{p}}\right)}{a^{3}}\right)^{\frac{1}{2}}
$$


We adopt units where $a=M_{\mathrm{s}}+M_{\mathrm{p}}=\Omega_{\mathrm{p}}=1$. The equations of motion for the particle with Cartesian coordinates $(x, y)$, whose origin is at the center of mass, are then

$$
\ddot{x}-2 \dot{y}-x+\frac{\mu[x-(1-\mu)]}{\left([x-(1-\mu)]^{2}+y^{2}\right)^{\frac{3}{2}}}+\frac{(1-\mu)(x+\mu)}{\left[(x+\mu)^{2}+y^{2}\right]^{\frac{3}{2}}}=0
$$

and

$$
\ddot{y}+2 \dot{x}-y+\frac{\mu y}{\left([x-(1-\mu)]^{2}+y^{2}\right)^{\frac{3}{2}}}+\frac{(1-\mu) y}{\left[(x+\mu)^{2}+y^{2}\right]^{\frac{3}{2}}}=0 .
$$

Following the standard procedure to obtain the Hill equations, we transform these equations to a coordinate system centered on the planet and rescale the coordinates. The scaling is chosen so that the new coordinates scale with the Hill radius, see equation (1). We make the change of variables to rescaled radius $R$ defined by

$$
\boldsymbol{R}=\left(\boldsymbol{r}-\boldsymbol{r}_{\mathrm{p}-\mathrm{cm}}\right) /\left(a \mu^{\frac{1}{3}}\right),
$$

where $\boldsymbol{r}_{\mathrm{p}-\mathrm{cm}}$ is the displacement of the planet from the center of mass. The equation of motion (9) is then

$$
\ddot{\boldsymbol{R}}+2 \hat{\boldsymbol{e}}_{z} \times \dot{\boldsymbol{R}}=-\nabla \Phi
$$

where $\Phi$ is the potential in this new frame that we determine in Section 3.2 We let $X=1-\mu+\mu^{\frac{1}{3}} x$ and $Y=\mu^{\frac{1}{3}} y$. Since $\mu$ is small, we consider here only the terms to lowest order in $\mu$. The equations to order $\mu^{\frac{1}{3}}$ become

$$
-3 X+\frac{X}{\left(X^{2}+Y^{2}\right)^{\frac{3}{2}}}-2 \dot{Y}+\ddot{X}=0
$$

and

$$
\frac{Y}{\left(X^{2}+Y^{2}\right)^{\frac{3}{2}}}+2 \dot{X}+\ddot{Y}=0 .
$$

In Section 3.4 we consider the higher order terms in $\mu$ that have been neglected here.

\subsection{The Potential in the Hill Approximation}

The terms in equations (16) and (17) that are functions of $X$ and $Y$ are expressed as the potential gradients

$$
\frac{\partial \Phi}{\partial X}=-3 X+\frac{X}{\left(X^{2}+Y^{2}\right)^{\frac{3}{2}}}
$$

and

$$
\frac{\partial \Phi}{\partial Y}=\frac{Y}{\left(X^{2}+Y^{2}\right)^{\frac{3}{2}}}
$$

Integrating these, we find the potential in the Hill approximation to be

$$
\Phi=-\frac{1}{\left(X^{2}+Y^{2}\right)^{\frac{1}{2}}}-\frac{3}{2} X^{2} .
$$

In polar coordinates centered on the planet, so that $X=R \cos \theta$ and $Y=R \sin \theta$, the potential is given by

$$
\begin{aligned}
\Phi & =-\frac{1}{R}-\frac{3}{2} R^{2} \cos ^{2} \theta \\
& =-\frac{1}{R}-\frac{3}{4} R^{2}-\frac{3}{4} R^{2} \cos 2 \theta .
\end{aligned}
$$

The first term is a point mass potential of the planet. The other two terms are due to the rotation of the frame and the gravitational effects of the star.

In dimensional form, the potential is given by

$$
\phi_{\mathrm{H}}=-\Omega_{\mathrm{p}}^{2} a^{2} \mu\left(\frac{a}{r}+\frac{3}{4 \mu}\left(\frac{r}{a}\right)^{2}(1+\cos 2 \theta)\right),
$$

which is valid for $r \sim O\left(\mu^{1 / 3} a\right)$.

\subsection{Angular Velocity in the Hill Approximation}

From the $r$-component of the equation of motion (9) with $\phi=\phi_{\mathrm{H}}$, we have that

$$
-\frac{u_{\theta}^{2}}{r}-2 \Omega_{\mathrm{p}} r\left(\Omega-\Omega_{\mathrm{p}}\right)=-\frac{\partial \phi_{\mathrm{H}}}{\partial r}
$$

We substitute the axisymmetric terms in the potential of equation (22) and the velocity in the $\theta$-direction, $u_{\theta}=r\left(\Omega-\Omega_{\mathrm{p}}\right)$, to find

$$
\Omega^{2}=\frac{G M_{\mathrm{p}}}{r^{3}}-\frac{1}{2} \Omega_{\mathrm{p}}^{2},
$$



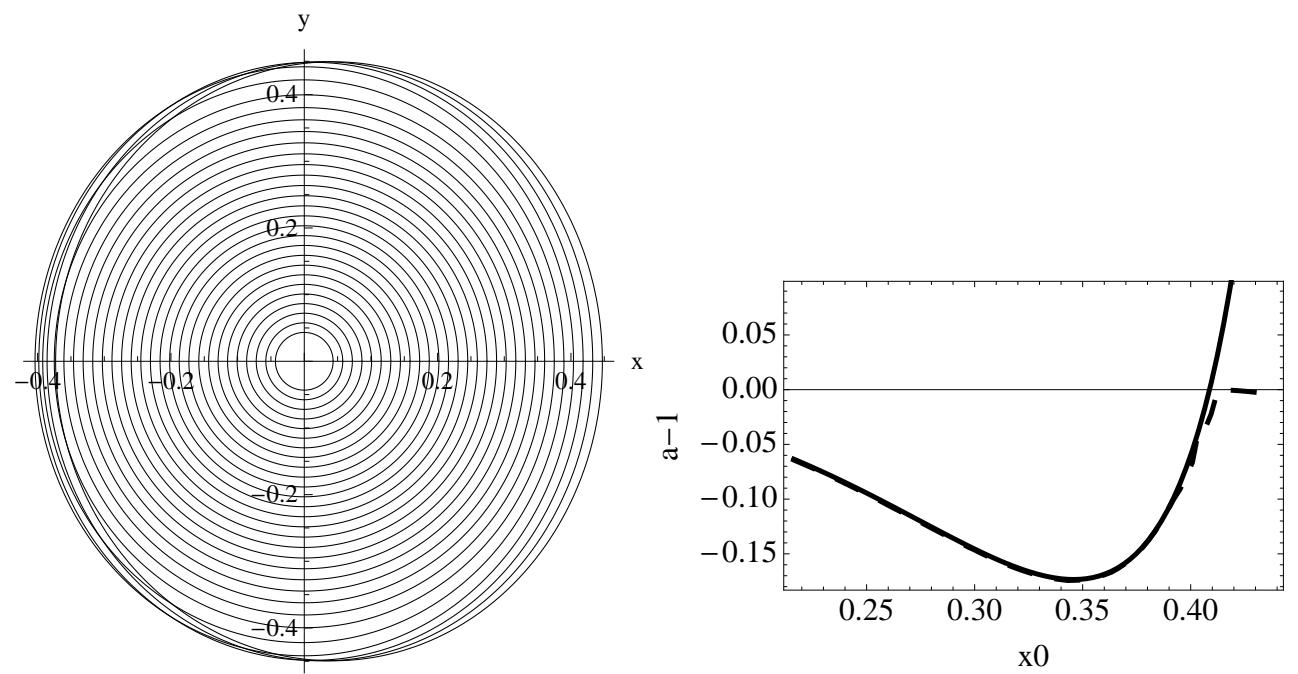

Figure 1. Left: Periodic orbits about a planet in the Hill approximation. The $x$ and $y$ coordinates are in units of the Hill radius $r_{\mathrm{H}}$. At a distance of about $0.4 r_{\mathrm{H}}$ from the planet, the orbits begin to cross. Right: Stability parameter (Henon 1965) $a-1$ plotted against $x_{0}$, the $x$ coordinate in units of $r_{\mathrm{H}}$ where the orbit crosses the positive $x$-axis. For $a-1$ negative (positive) the orbits are dynamically stable (unstable). The dashed line is for periodic orbits that eventually intersect each other at larger $x_{0}$, as occurs in the left panel. The solid line tracks the stability of periodic orbits that remain symmetric about the $y$-axis for larger $x_{0}$. Notice that for $x_{0} \simeq 0.4 r_{\mathrm{H}}$, where orbits begin to cross in the left panel, there is a bifurcation in stability properties. At larger radii, $y$-symmetric orbits remain nonintersecting, but are unstable, while the intersecting orbits are marginally stable.

where $r$ is the (unscaled) distance from the planet. There are corrections to this result due to nonlinear effects of nonaxisymmetric terms. The angular velocity about the planet, $\Omega$, is less than Keplerian because of the effects of the star.

\subsection{Crossing and Unstable Orbits}

We numerically solved equations (16) and (17) to find the trajectories of particles in this potential. We found closed periodic particle orbits in the frame of the planet, along the lines of Paczynski (1977), but in the Hill approximation. We chose the initial position for the particle to be on the $x$-axis. The initial $y$-velocity is determined so that the particle has zero velocity in the $x$ direction when it again crosses the $x$-axis on the other side of the central planet. We find that the neighboring particle orbits first intersect for the initial position on the $x$-axis of $x=0.410 r_{\mathrm{H}}$ (or $X=0.294$ ), as shown in the left panel of Fig. 1 This maximum radius of nonintersecting orbits, $r_{\max } \simeq 0.410 r_{\mathrm{H}}$, is considered to represent a maximum disc radius for a cold disc, as discussed by Paczynski (1977).

The orbits in in the left panel Fig. 1 are asymmetric (lopsided) with respect to the y-axis in that the intersections occur for $x<0$. On the other hand, the $m=2$ Hill potential is symmetric about the y-axis. A more detailed analysis shows that there is another set of orbits for which the intersections occur for $x>0$. In addition, there is a third set of orbits that remain symmetric about the y-axis and nonintersecting for $r>r_{\max }$. However, this set of orbits is dynamically unstable for $r>r_{\max }$. That is, there is a bifurcation that occurs at $r=r_{\max }$, as seen in the right panel of Fig. 1 Beyond this radius, there continue to exist y-symmetric nonintersecting orbits, but they are unstable. There are stable orbits beyond this radius, but they intersect with neighboring orbits. In any case, this critical radius of $r_{\max }=0.410 r_{\mathrm{H}}$ is the limiting radius for orbits that represent a cold, steady-state disc.

To test the validity the Hill approximation, we also solved the equations of motion for the full gravitational potential in the frame of the planet. We determined the particle orbits in the same way as described above. We found that the orbits first intersect at a radius of $0.412 r_{\mathrm{H}}$ with a mass ratio $\mu=0.01$. The Hill approximation is then reasonably accurate for these purposes, even for relatively large mass planets.

\subsection{Higher Order terms}

By including terms of $\mathrm{O}\left(\mu^{\frac{2}{3}}\right)$ and $\mathrm{O}(\mu)$ in equations (16) and (17), we obtain the dimensionless potential

$$
\Phi=-\frac{1}{R}-\frac{3}{4} R^{2}-\frac{3}{4} R^{2} \cos 2 \theta+\frac{\mu^{\frac{1}{3}}}{8} R^{3}(3 \cos \theta-5 \cos 3 \theta)-\frac{\mu^{\frac{2}{3}}}{64} R^{4}(9+20 \cos 2 \theta+35 \cos 4 \theta) .
$$

These higher order terms in $\mu^{1 / 3}$ introduce additional angular dependencies. 


\section{DISC FLUID EQUATIONS}

The steady-state two-dimensional equations of motion for a fluid disc in the corotating frame are

$$
\nabla \cdot(\Sigma \mathbf{u})=0
$$

and

$$
(\boldsymbol{u} \cdot \boldsymbol{\nabla}) \boldsymbol{u}+2 \boldsymbol{\Omega}_{\mathrm{p}} \times \boldsymbol{u}=-\nabla \boldsymbol{\phi}-\frac{1}{\Sigma} \nabla p+\boldsymbol{f}_{\mathrm{v}},
$$

where $\boldsymbol{u}$ is the fluid velocity and $\boldsymbol{f}_{\mathrm{v}}$ is the force per unit disc mass that represents the effects of turbulent viscosity. Quantity $p$ is the two-dimensional (vertically integrated) pressure. We consider a cylindrical coordinate system $(r, \theta)$ centered on the planet. The azimuthal viscous force due to shear is given by

$$
f_{r, \theta}=\frac{1}{r^{2} \Sigma} \frac{\partial}{\partial r}\left(\nu \Sigma r^{3} \frac{d \Omega}{d r}\right)
$$

where $\Sigma$ is the surface density and $\nu$ is the kinematic turbulent viscosity. The potential $\phi$ is given by equation (10).

We rescale the variables by $\mu^{1 / 3}$ as we did for the ballistic case in equation (14). We rescale the velocity and radius as $\boldsymbol{U}=\boldsymbol{u} /\left(\mu^{\frac{1}{3}} a \Omega_{\mathrm{p}}\right)$ and $R=r /\left(\mu^{1 / 3} a\right)$, respectively, and obtain

$$
\tilde{\nabla} \cdot(\Sigma \mathbf{U})=0
$$

and

$$
(\boldsymbol{U} \cdot \tilde{\nabla}) \boldsymbol{U}+2 \tilde{\Omega}_{\mathrm{p}} \times \boldsymbol{U}=-\tilde{\nabla} \Phi-\frac{1}{\Sigma} \tilde{\nabla} P+\boldsymbol{F}_{\mathrm{v}}
$$

where

$$
\begin{gathered}
\boldsymbol{F}_{\mathrm{v}}=\boldsymbol{f}_{\mathrm{v}} /\left(\mu^{1 / 3} a^{2} \Omega_{\mathrm{p}}\right), \\
\tilde{\boldsymbol{\nabla}}=\left(\mu^{1 / 3} a\right) \boldsymbol{\nabla}, \\
P=p /\left(\mu^{2 / 3} a^{2} \Omega_{\mathrm{p}}^{2}\right), \\
\tilde{\boldsymbol{\Omega}}=\boldsymbol{\Omega} / \Omega_{\mathrm{p}},
\end{gathered}
$$

and

$$
\Phi=\phi /\left(\mu^{2 / 3} a^{2} \Omega_{\mathrm{p}}^{2}\right)
$$

The potential, $\Phi$, is given by equation (25) and is independent of $\mu$ to lowest order, the Hill approximation. The rescaled viscous shear force is

$$
F_{r, \theta}=\frac{1}{R^{2} \Sigma} \frac{\partial}{\partial R}\left(\alpha R^{5} \Sigma\left(\frac{H}{r}\right)^{2} \tilde{\Omega} \frac{d \tilde{\Omega}}{d R}\right),
$$

where we used the $\alpha$-prescription for the viscosity so that

$$
\nu=\alpha\left(\frac{H}{r}\right)^{2} r^{2} \Omega=\mu^{2 / 3} \alpha\left(\frac{H}{r}\right)^{2} R^{2} \tilde{\Omega} a^{2} \Omega_{\mathrm{p}}
$$

(Shakura \& Sunvaev 1973). We have left the disc aspect ratio about the planet in the unscaled form $H / r$ because it is invariant to scaling. We have not rescaled the surface density because doing so would have no effect on the velocity field, since we neglect disc self-gravity. Notice that $F_{r, \theta}$ is independent of the mass ratio $\mu$. This is also true of all other components of the viscous force. The rescaled pressure is given by

$$
P=\Sigma\left(\frac{H}{r}\right)^{2} R^{2} \tilde{\Omega}^{2}
$$

which is independent of $\mu$. Thus, the transformed equations (29) and (30) are of the same form as the original equations (26) and (27) and are independent of $\mu$ in the Hill approximation. This result also holds in an obvious extension to three dimensions.

The rescaled circumplanetary disc equations are then very similar to the circumstellar disc equations previously analysed for close binary stars with order unity mass ratio. One difference is the form of the potential, which has a single nonaxisymmetric azimuthal number $m=2$ in the Hill approximation. However, this is the dominant tidal term in close binaries. Consider a disc in binary that orbits star 1 and is tidally perturbed by star 2 . At a small distance $r \ll a$ from star 1 , the potential terms due to the star 1 and the $m=2$ tidal component due to star 2 is given by $\phi=-G M_{1} / r-3 G M_{2} \cos (2 \theta) r^{2} /\left(4 a^{3}\right)$. This potential is similar (within a factor of 2) to the rescaled potential of equation (21) with $G=1, a=1$, and masses $M_{1}=M_{2}=1 / 2$. In particular, the ratio of the $m=2$ tidal to central potentials is the same for both cases. Therefore, the disc flow equations for a planet in the Hill approximation are similar to those for a binary star system with unity mass ratio, for a given value of $H / r$ and $\alpha$. 


\section{RESONANCES}

Resonances occur in the disc where the forcing frequency matches a natural frequency in the disc. Angular momentum can be transferred to the star from the disc by the tidal torques that are exerted at the Lindblad resonances within the disc (Goldreich \& Tremaine 1979). At such resonances, rotationally modified pressure waves are launched. Torques are exerted on the disc at radii where the waves damp. Resonance torques could play a role in truncating the disc. In this section we consider whether circular, eccentric, or vertical resonances could lie within the disc. We only consider nearly circular orbits in this analysis. We find this approximation holds well out to the radius where orbits begin to cross (Section 3.4). Since we expect the disc to be truncated inside or at this radius, we should find all of the resonances present in the disc in this approximation.

However, for a disc as warm as suggested during the $\mathrm{T}$ Tauri accretion phase, $H / r \sim 0.3$ (equation (4)), some gas might extend beyond the orbit crossing radius and off-resonant forcing could play a role. Off-resonant forcing is possible because for $m$ of order unity, the resonance width scales as $(H / r)^{2 / 3} r_{\mathrm{H}}$. With such a large width, the resonance could overlap with the disc, even though the exact resonance location does not lie within the main body of the disc, as has been investigated in the binary star case by Savoniie. Papaloizou \& Lin (1994).

\subsection{Circular Resonances}

We apply the angular velocity of the disc in the Hill approximation given in equation (24) to the Lindblad resonance condition Goldreich \& Tremaine (1979). The circular Lindblad resonances then occur where

$$
-m^{2}\left(\Omega-\Omega_{\mathrm{p}}\right)^{2}+4 \Omega\left(\Omega+\frac{r}{2} \frac{d \Omega}{d r}\right)=0 .
$$

In the Hill approximation we have only the $m=2$ term of the potential. We find that the only positions inside the orbit crossing radius is for the $m=1$ term which is at $R=0$. For a cold disc, the resonance width is very small and the tidal forcing is very weak near the disc center. For a warm disc, stronger resonant excitation is possible. At higher order in $\mu^{1 / 3}$, other $m$-values are present (equation 25). However, these higher order resonances also fail to lie inside the orbit crossing radius.

\subsection{Eccentric Resonances}

If the orbit of the planet is slightly eccentric with eccentricity $e$, the tidal forcing can be decomposed into a series or rigidly rotating potentials at various frequencies $\ell \Omega_{\mathrm{p}}$ for integer $\ell$ (Goldreich \& Tremaine 1979). The eccentric Lindblad resonances then occur where

$$
-\left(m \Omega-\ell \Omega_{\mathrm{p}}\right)^{2}+4 \Omega\left(\Omega+\frac{r}{2} \frac{d \Omega}{d r}\right)=0
$$

For $m=2$, the lowest order resonance that lies within the orbit crossing radius has $\ell=7$. The resonant torque scales as $e^{2|l-m|}$, where $m$ is the azimuthal wavenumber of the tidal potential. Consequently, the torque would scale as $e^{10}$, which suggests that the torque would be quite weak for modest eccentricities. For such a resonance to be able to overcome the effects of disc turbulent viscosity and truncate the disc, we roughly require $\alpha \lesssim(r / H)^{2} e^{10}$ (see, Artvmowicz \& Lubow 1994). Consequently, the disc viscosity would need to be very small.

\subsection{Vertical Resonances}

There is also a set of resonances associated with the vertical disc motions. For each $m$, the vertical disc resonance lies closer to the planet than the corresponding horizontal (coplanar) Lindblad resonance, although the torque it produces is a factor of $(H / r)^{2}$ smaller than the corresponding horizontal resonance. The vertical resonance generates horizontally propagating waves which travel towards the planet at the centre of the disc. The wave generation can transport angular momentum to the orbital motion of the planet-star system. The vertical resonances occur where

$$
m^{2}\left(\Omega-\Omega_{\mathrm{p}}\right)^{2}=(1+\Gamma)\left(\Omega_{\mathrm{p}}^{2}+\frac{G M_{p}}{r^{3}}\right)
$$

where $\Gamma$ is the effective adiabatic index (Lubow 1981). We solve this equation with $\Gamma=1.4$ and find the $m=2$ resonance occurs at $r / r_{\mathrm{H}}=0.49$, which is somewhat outside the orbit crossing radius. Resonances at higher $m$-values occur further away from the planet. The analysis in subsequent sections of this paper considers only one- and two-dimensional models that are not capable of finding vertical resonances. 


\section{VISCOUS DISC MODEL}

We consider the effect of the tidal perturbation on a circumplanetary accretion disc due to the presence of the star by following the work of Papaloizou \& Pringle (1977). The tidal torque transfers angular momentum from the disc to the binary orbit, allowing material to accrete on to the central object (Börner et al. 1973; Lin \& Pringle 1976). The disc attempts to expand by viscous forces, but tidal torques dominate the transport process only in the outer parts of the disc. In this approach, the disc pressure forces are ignored. Consequently, this approach does not include the effects of off-resonant waves that could be present. It is more accurate for cool discs, $H / r<0.1$, where the effects of waves are likely less important. Such cool circumplanetary discs could arise at late stages of disc evolution where the accretion rates are lower as the disc disperses.

\subsection{Linearised Velocity}

We consider linearised equations for the tidal disturbances about the circular motions around the planet and initially include only gravitational and centrifugal forces. The unperturbed gas orbits with velocity $\boldsymbol{u}_{\mathbf{0}}=\left(0, r\left(\Omega-\Omega_{\mathrm{p}}\right)\right)$ in the corotating frame centered on the planet, where $\Omega$ is given by equation (24). We consider perturbations to this flow by the presence of the star. We denote the perturbed velocity as $\boldsymbol{u}_{\mathbf{1}}$, where $u_{1} \ll u_{0}$ and $\boldsymbol{u}_{\mathbf{1}}=\left(u_{r}, u_{\theta}\right)$. We follow the approach of Papaloizou \& Pringle (1977), but adopt the corotating frame with the Hill approximation. The linearised equations of motion based on equation (27) are

$$
\left(\Omega-\Omega_{\mathrm{p}}\right) \frac{\partial u_{r}}{\partial \theta}-2 \Omega u_{\theta}=f_{r}
$$

and

$$
\left(\Omega-\Omega_{\mathrm{p}}\right) \frac{\partial u_{\theta}}{\partial \theta}+2 B u_{r}=f_{\theta}
$$

where

$$
B=\frac{r}{2} \frac{d \Omega}{d r}+\Omega
$$

The force components are obtained from the negative gradient of potential $\phi_{\mathrm{H}}$ in equation (22).

Since only $m=2$ disturbances are involved in the Hill approximation, we represent

$$
u_{r}, u_{\theta}, f_{R}, f_{\theta} \propto \exp (2 i \theta)
$$

and obtain

$$
u_{r}=\frac{i\left(\Omega-\Omega_{\mathrm{p}}\right) f_{r}+\Omega f_{\theta}}{2\left[-\left(\Omega-\Omega_{\mathrm{p}}\right)^{2}+B \Omega\right]}
$$

and

$$
u_{\theta}=\frac{-B f_{r}+i\left(\Omega-\Omega_{\mathrm{p}}\right) f_{\theta}}{2\left[-\left(\Omega-\Omega_{\mathrm{p}}\right)^{2}+B \Omega\right]} .
$$

\subsection{Dissipation}

We now consider the additional dissipation in the disc due to tidal perturbations when we introduce a small amount of dissipation into the flow. The two modes of dissipation in a Newtonian fluid are the shear viscosity, $\nu$, and the bulk viscosity, $\zeta$. The dissipation due to the bulk viscosity per unit radius is

$$
D_{1}=\Sigma \zeta \int_{0}^{2 \pi}(\boldsymbol{\nabla} \cdot \boldsymbol{u})^{2} d \theta
$$

which we can evaluate using equations (46) and (47). The dissipation due to the shear viscosity is

$$
D_{2}=2 \Sigma \nu \int_{0}^{2 \pi} E_{i j} E_{i j} d \theta
$$

where $E_{i j}=e_{i j}-1 / 3 \delta_{i j} \boldsymbol{\nabla} \cdot \boldsymbol{u}$ and $e_{i j}$ is the rate of strain tensor which is given by

$$
e=\left(\begin{array}{cc}
\frac{\partial u_{r}}{\partial r} & \frac{1}{2}\left[r \frac{\partial\left(u_{\theta} / r\right)}{\partial r}+\frac{1}{r} \frac{\partial u_{\theta}}{\partial \theta}\right] \\
\frac{1}{2}\left[r \frac{\partial\left(u_{\theta} / r\right)}{\partial r}+\frac{1}{r} \frac{\partial u_{\theta}}{\partial \theta}\right] & \frac{1}{r} \frac{\partial u_{\theta}}{\partial \theta}+\frac{u_{r}}{r}
\end{array}\right) .
$$



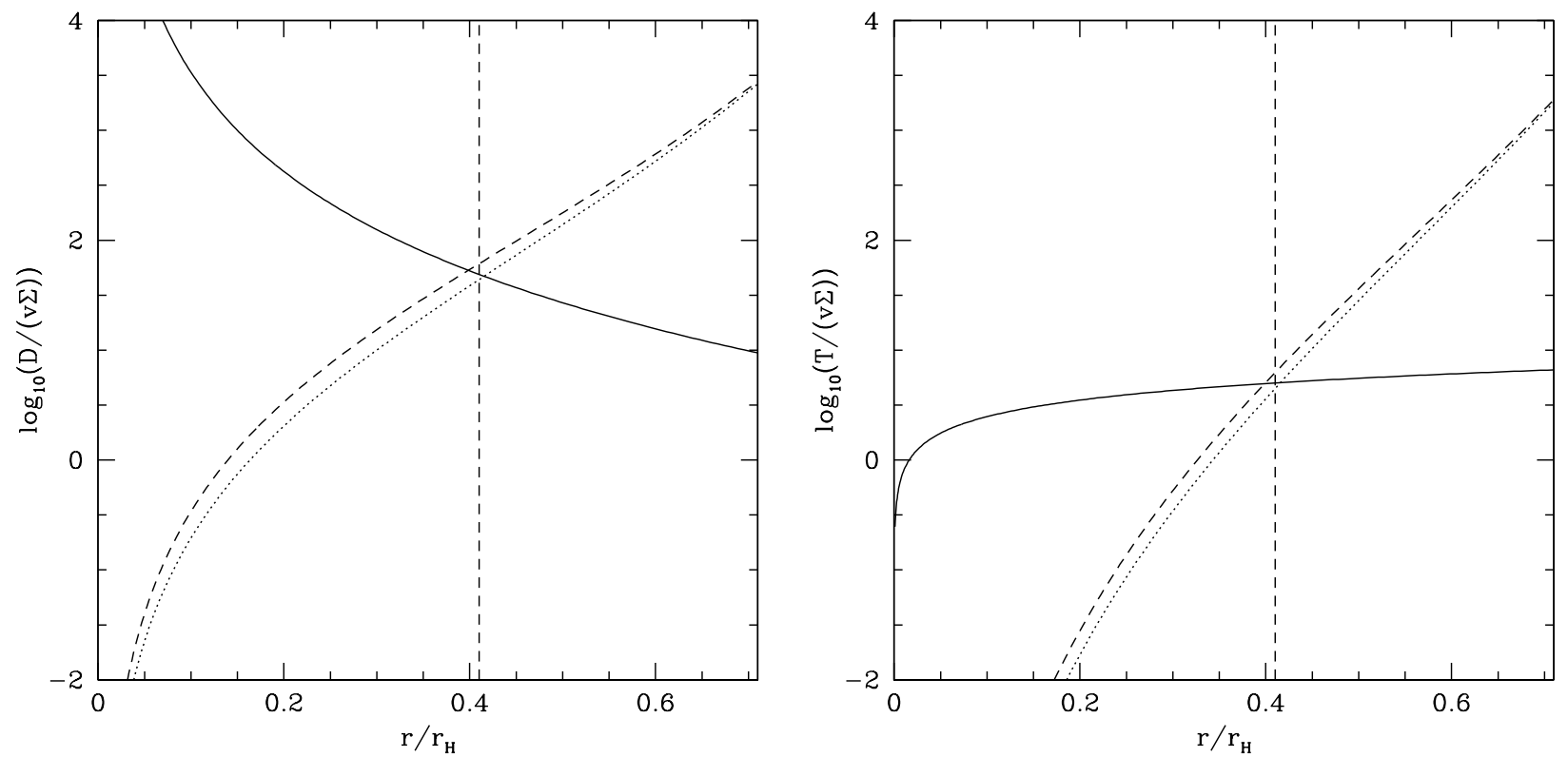

Figure 2. Left: The dissipation in the disc. The solid line is $D_{0} /(\nu \Sigma)$, the dashed line is $D_{1} /(\zeta \Sigma)$ and the dotted line is $D_{2} /(\nu \Sigma)$. Right: The torque acting on the disc per unit surface density, per unit viscosity. The solid line is for the viscous torque in the disc, $T_{0} /(\nu \Sigma)$, the dotted line is the torque $T_{1} /(\zeta \Sigma)$ and the dashed line is $T_{2} /(\nu \Sigma)$. For both plots the vertical dotted lines show where the particle orbits cross.

\subsection{Torque on the disc}

From the dissipation we can find the torque on the disc due to the tidal perturbation. The rate of working per unit surface area of the disc by a torque, $T$, is

$$
\begin{aligned}
D & =\Omega \frac{\partial T}{\partial r} d r \frac{1}{4 \pi r d r}=\frac{\Omega}{4 \pi r} \frac{\partial T}{\partial r} \\
& =\frac{1}{4 \pi r}\left[\frac{\partial(T \Omega)}{\partial r}-T \Omega^{\prime}\right]
\end{aligned}
$$

where $\Omega^{\prime}=d \Omega / d r$. The first term is the rate of convection of energy over the whole disc. Its value depends only on the boundary conditions. We find the torque on the disc to be

$$
T_{i}=\frac{4 \pi r D_{i}}{\Omega^{\prime}},
$$

where $i=0,1,2$ (Frank, King \& Raine 2002). The torque $T_{0}$ is defined as the torque on an accretion disc without a companion. This has a viscous torque of

$$
T_{0}=2 \pi r \nu \Sigma r^{2} \Omega^{\prime}
$$

and so the dissipation is

$$
D_{0}=\frac{1}{2} r^{2}\left(\Omega^{\prime}\right)^{2} \nu \Sigma
$$

In the left panel of Fig. 2 we plot the three scaled dissipations, $D_{0} /(\nu \Sigma), D_{1} /(\zeta \Sigma)$ and $D_{2} /(\nu \Sigma)$ as functions of the radius in the disc. This is similar to Fig. 1 in Papaloizou \& Pringle (1977), but we use the Hill approximation. We see that the magnitude of the dissipation from the internal viscous torques, $D_{0} /(\nu \Sigma)$, and the tidal dissipation, $D_{2} /(\nu \Sigma)$, are equal very close to, but just inside, the radius in the disc where the particle orbits begin to cross. In the right panel of Fig. 2 we plot the scaled torques on the disc. The tidal torque in the disc starts to dominate the viscous torque just inside of the radius where the particle orbits cross.

However, outside of the radius where the particle orbits cross, pressure and nonlinear effects cannot be neglected. Therefore, this linear solution is not valid beyond the orbit crossing radius. 


\subsection{One-Dimensional Simulations}

We use a one-dimensional (radius only) model of an accretion disc subject to a tidal torque in order to determine the surface density evolution. The governing equation for the surface density of a flat accretion disc centered on the planet is

$$
\frac{\partial \Sigma}{\partial t}=\frac{1}{r} \frac{\partial}{\partial r}\left[\frac{1}{\left[r^{2} \Omega\right]^{\prime}} \frac{\partial}{\partial r}\left(\nu \Sigma r^{3}\left(-\Omega^{\prime}\right)\right)-\frac{\Sigma r}{\left[r^{2} \Omega\right]^{\prime}} \frac{d T_{\mathrm{gr}}}{d M}\right]+S(r)
$$

(Pringle 1981), where ${ }^{\prime}=d / d r$ and the angular velocity is given in equation (24). In the previous section, we found the tidal torque on the disc. However, this is only valid up to the position in the disc where the orbits cross. We adopt a torque function per unit disc mass on the disc of the form

$$
\frac{d T_{\mathrm{gr}}}{d M}=-T_{3}(r)\left(\frac{r}{r_{\mathrm{H}}}\right)^{g}
$$

where $g$ is a constant. To model the tidal effects of orbit crossings, we select the torque parameters so that the torque acts in the region where the particle orbits begin to cross. We choose $g=4$ so that the disc is truncated quickly. The form of $T_{3}$ controls where the disc is truncated. We choose

$$
T_{3}(r)= \begin{cases}0.5 r_{\mathrm{H}}^{2} \Omega_{\mathrm{p}}^{2} & r \geqslant 0.4 r_{\mathrm{H}} \\ 0 & r<0.4 r_{\mathrm{H}}\end{cases}
$$

To model the effects of the inflowing circumstellar gas, we model gas injection at some radius $r_{\text {inj }}$ over a narrow region of radial width $2 w$ at a steady rate $\dot{M}_{\text {inj }}$ with the local Keplerian speed. Function $S(r)$ describes the mass injection that we take to be

$$
S(r)=\frac{\dot{M}_{\text {inj }}}{2 \pi r_{\text {inj }}} \frac{H\left(\left(r-r_{\text {inj }}\right) / w\right)}{2 w},
$$

where $H(x)$ is unity for $|x|<1$ and zero otherwise. We adopt a width $w=0.0046\left(r_{\mathrm{inj}} / r_{\mathrm{H}}\right)^{\frac{1}{2}} r_{\mathrm{H}}$.

We solve this equation numerically on a fixed mesh that is uniform $r^{\frac{1}{2}}$ with 200 grid points (like Martin et al. 2007). We choose zero torque boundary conditions at the inner boundary $r_{\text {in }}=10^{-3} r_{\mathrm{H}}$ and the outer boundary $r_{\mathrm{out}}=0.9 r_{\mathrm{H}}$. The inner boundary allows the material there to be accreted by the planet. Its position has been chosen so that it is about equal to the radius of a Jupiter planet at a distance of $5 \mathrm{AU}$ from a central solar mass star. With the tidal torque acting, the outer boundary is far enough out that it does not affect the evolution because the tidal torque prevents the mass from reaching the outer regions. However, if there is no tidal torque, the disc extends as far as it can and the outer boundary will affect the mass in the disc because mass is removed there. The viscous torque is given in equation (53) and we take $\Sigma=0$ at the boundaries to have zero torques there. We initially take the surface density to be a constant but very small value and allow it to build up by mass accretion at a radius $r_{\text {inj }}$.

We set the accretion rate on to the disc to be $\dot{M}=1.78 \times 10^{-8} M_{\mathrm{s}} \Omega_{\mathrm{p}}$. For a Jupiter mass planet orbiting a solar mass star at a radius of $5 \mathrm{AU}$ this corresponds an accretion rate of $\dot{M}=10^{-8} \mathrm{M}_{\odot} \mathrm{yr}^{-1}$. We start with a disc of nearly zero mass. We note that changing the accretion rate does not change the results in Fig. 3, only the amount of mass in the disc. We parametrise the viscosity with the $\alpha$-prescription in equation (5). For a Keplerian disc with $\alpha=10^{-3}$ and a constant disc aspect ratio $H / r=0.3$, we have gas kinematic turbulent viscosity

$$
\nu=1.56 \times 10^{-4}\left(\frac{r}{r_{\mathrm{H}}}\right)^{\frac{1}{2}} r_{\mathrm{H}}^{2} \Omega_{\mathrm{p}} .
$$

Disc mass builds up as mass is injected. We ran the numerical code until it reached a steady state. In Fig. 3 we plot the surface density evolution of the disc for different injection radii. The surface density in each of the plots increases as time goes on. The top left plot shows the evolution without the tidal torque and an injection radius $r_{\mathrm{inj}}=0.2 r_{\mathrm{H}}$. We see that the disc spreads out as far as it can out to the outer boundary, where mass is removed. All the other three plots in Fig. 3 include the tidal torque and we vary the position that mass is added from $r_{\text {inj }}=0.1 r_{\mathrm{H}}$ (top right), $0.2 r_{\mathrm{H}}$ (bottom left) and $0.3 r_{\mathrm{H}}$ (bottom right). With the tidal torque acting, the disc cannot expand out to the outer boundary, it becomes truncated well inside that boundary. The position of the injection of the mass does not affect the outer boundary of the disc, it only mildly modifies the surface density profile of the steady state disc.

\subsection{One-Dimensional Analytic Solutions}

In this section we find steady-state analytical solutions for the surface density of a circumplanetary accretion disc. Our approach is similar to that of Canup \& Ward (2002) and Ward \& Canup (2010), but we include the strong tidal torques near the orbit crossing radius. There are three regions in the disc, as shown in Fig. 4 Inside of the radius where mass is added, $r_{\text {inj }}$, the disc acts as a normal accretion disc. Outside of this radius, the disc acts as a decretion disc in terms of the density profile, although gas does not actually flow outward, as shown below. Instead, this region acts as mass reservoir. However, material in this region is subject to exchange with the interior region $r<r_{\text {inj }}$ by means of turbulent diffusion. A power law 

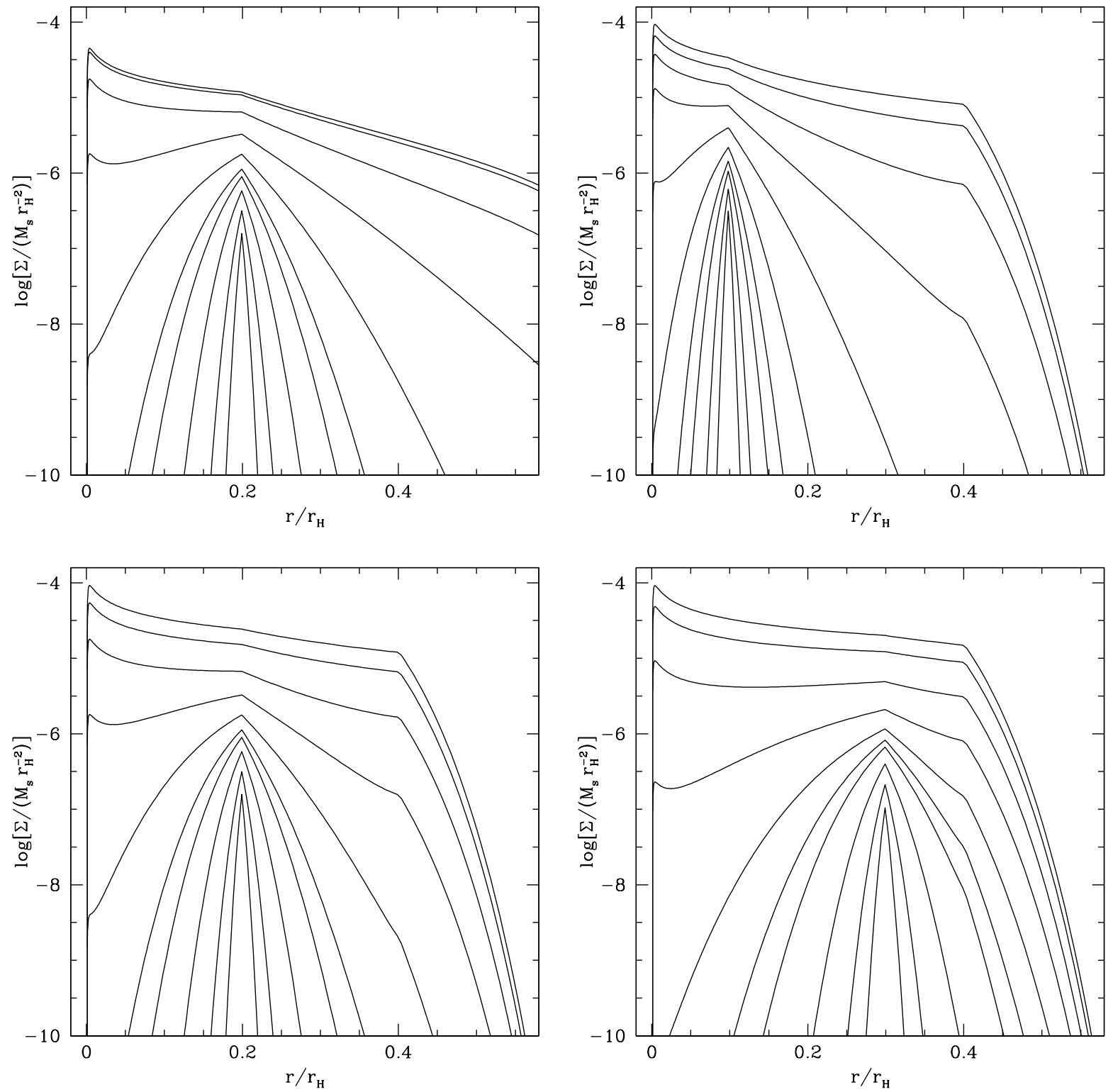

Figure 3. Top left: The evolution of the disc without the tidal torque with $r_{\text {inj }}=0.2 r_{\mathrm{H}}$. The remaining plots show the evolution of the disc with the binary torque with $r_{\mathrm{inj}}=0.1 r_{\mathrm{H}}$ (top right), $r_{\mathrm{inj}}=0.2 r_{\mathrm{H}}$ (bottom left) and $r_{\mathrm{inj}}=0.3 r_{\mathrm{H}}$ (bottom right). The surface density increases in time. The times plotted, in order of increasing surface density in the plots, $t=0.01,0.05,0.18,0.42,0.67,1.63,5.50$, $20.96,82.79$ and $330.13 P$ where $P$ is the orbital period of the planet, $P=2 \pi / \Omega_{\mathrm{p}}$.

torque is applied only in the outer parts of the disc in $r>r_{\text {trunc. }}$. We assume for simplicity that the disc is in exact Keplerian rotation about the planet with angular speed $\Omega$ in the inertial frame.

The accretion rate through the disc is

$$
\dot{M}=2 \pi r u_{r} \Sigma .
$$

Notice that $\dot{M}$ is negative for accretion $\left(u_{r}<0\right)$. Mass is injected at a radius $r=r_{\text {inj }}$ with rate $\dot{M}_{\text {inj }}>0$, that is

$$
\frac{d \dot{M}}{d r}=\dot{M}_{\mathrm{inj}} \delta\left(r-r_{\mathrm{inj}}\right)
$$

and the angular momentum equation is

$$
\frac{d\left(\dot{M} r^{2} \Omega\right)}{d r}=\frac{d}{d r}\left(T_{\nu}+T_{\mathrm{gr}}\right)+\dot{M}_{\mathrm{inj}} r_{\mathrm{inj}}^{2} \Omega_{\mathrm{inj}} \delta\left(r-r_{\mathrm{inj}}\right),
$$

where the viscous torque per unit radius (with equation 53) is given by

$$
T_{\nu}=-3 \pi r^{2} \Omega \Sigma \nu
$$




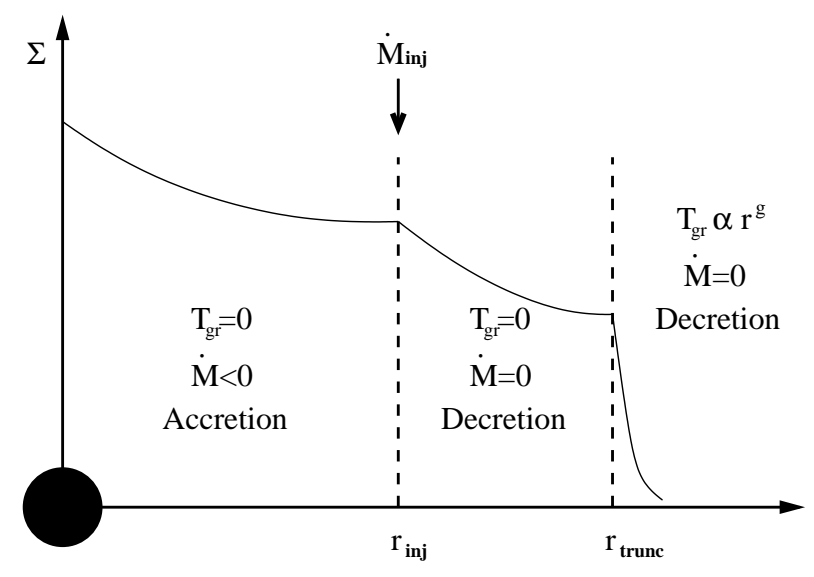

Figure 4. A sketch of the surface density about a planet in the three region model. In $r<r_{\text {inj }}$ there is an accretion disc and in $r>r_{\text {inj }}$ there is a decretion disc structure that acts as a mass reservoir. In this model, for $r<r_{\text {trunc }}$ there is no imposed gravitational torque and for $r>r_{\text {trunc }}$ a power law gravitational torque is applied.

for a Keplerian angular velocity where $\Omega_{\mathrm{inj}}=\Omega\left(r_{\mathrm{inj}}\right)$ and $T_{\mathrm{gr}}$ is the gravitational torque. Equation (62) is equivalent to equation (55) with the time derivative set to zero and injection width $w$ set to (nearly) zero.

In accordance with equation (56), the gravitational torque per unit radius on the disc is taken to be

$$
\frac{d T_{\mathrm{gr}}}{d r}=-2 \pi r \sum T_{3}(r)\left(\frac{r}{r_{\mathrm{H}}}\right)^{g}
$$

where $T_{3}$ is defined by equation (57). Integrating equation (61) we find

$$
\dot{M}= \begin{cases}-\dot{M}_{\mathrm{inj}}+C_{1} & r<r_{\mathrm{inj}} \\ C_{1} & r>r_{\mathrm{inj}},\end{cases}
$$

where $C_{1}$ is a constant. With the boundary condition that $\dot{M}=0$ at the disc outer edge, we see that $C_{1}=0$. So there is no accretion flow outside of the injection radius, all of the material that is injected at $r_{\text {inj }}$ flows inwards to be accreted on to the planet. We now integrate equation (62) to find

$$
\begin{array}{rlrl}
T_{\nu}+\dot{M}_{\mathrm{inj}} r^{2} \Omega+C_{2} & =0, & & r<r_{\mathrm{inj}} \\
T_{\nu}+\dot{M}_{\mathrm{inj}} r_{\mathrm{inj}}^{2} \Omega_{\mathrm{inj}}+C_{2}=0, & & r_{\mathrm{inj}}<r<r_{\mathrm{trunc}} \\
T_{\nu}+T_{\mathrm{gr}}+\dot{M}_{\mathrm{inj}} r_{\mathrm{inj}}^{2} \Omega_{\mathrm{inj}}+C_{2}=0, & & r>r_{\text {trunc }},
\end{array}
$$

where $C_{2}$ is a constant to be determined by the inner boundary condition. As in Section 6.4, we have chosen the gravitational torque to be zero in $r<r_{\text {trunc }}$.

In evaluating equation (66), we apply the form of kinematic viscosity given by equation (59), $\nu(r) \propto \sqrt{r}$, that holds for a constant $\alpha$ and constant $H / r$ circumplanetary disc. With the zero viscous torque inner boundary condition at an inner radius $r_{\mathrm{in}}$, equation (66) requires $C_{2}=-\dot{M}_{\mathrm{inj}} r_{\mathrm{in}}^{2} \Omega_{\mathrm{in}}$. We then obtain the standard viscous disc surface density in the inner parts of the disc $r<r_{\mathrm{inj}}$, designated by subscript 1 ,

$$
\Sigma_{1}(r)=\frac{\dot{M}_{\mathrm{inj}}}{3 \pi \nu(r)}\left[1-\left(\frac{r_{\mathrm{in}}}{r}\right)^{\frac{1}{2}}\right]
$$

(Pringle 1981). Therefore, $\Sigma_{1}(r) \propto 1 / \sqrt{r}$, for $r_{\text {inj }}>r \gg r_{\text {in }}$. In the other two regions of space where $r>r_{\text {inj, }}$, we assume that $r \gg r_{\text {in }}$, so that $C_{2}$ is very small compared to the other torques. For simplicity, we take $C_{2}=0$ in these regions.

In the region outside of where the mass is injected, but where no gravitational torque is applied, $r_{\mathrm{inj}}<r<r_{\text {trunc }}$, the surface density is

$$
\Sigma_{2}(r)=\Sigma_{1}\left(r_{\mathrm{inj}}\right)\left(\frac{r_{\mathrm{inj}}}{r}\right) .
$$

In the outermost region, $r \geqslant r_{\text {trunc }}$, the surface density is

$$
\Sigma_{3}=\Sigma_{2}\left(r_{\text {trunc }}\right)\left(\frac{r_{\text {trunc }}}{r}\right) \exp \left[-\frac{2\left(r^{1+g}-r_{\text {trunc }}^{1+g}\right) T_{3}\left(r_{\text {trunc }}\right)}{3(1+g) \nu_{\text {trunc }} \Omega_{\text {trunc }} r_{\text {trunc }} r_{\mathrm{H}}^{g}}\right],
$$

where $\nu_{\text {trunc }}=\nu\left(r_{\text {trunc }}\right)$ and $\Omega_{\text {trunc }}=\Omega\left(r_{\text {trunc }}\right)$. We now have the surface density for the whole disc with the equations for $\Sigma_{1}, \Sigma_{2}$ and $\Sigma_{3}$. We compared these solutions to the one-dimensional cases of Section 6.4 with the same values for the input parameters. These steady state analytic solutions agree well (better than 6\%) with the numerical solutions obtained at late times, plotted as the highest lines in the plots in Fig. 3. 


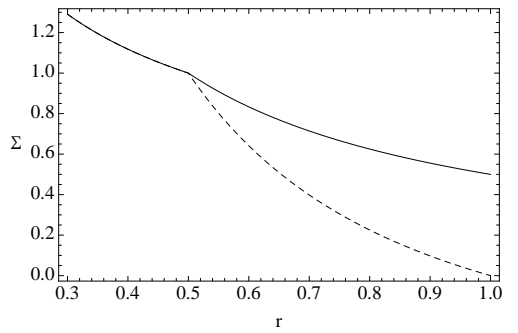

Figure 5. Surface density distributions for a disc with constant $\alpha$ and constant $H / r$ as a function of radius for cases where the mass is injected at radius $r=0.5$. The surface density is normalized by its value at the injection radius. The dashed line is for a model that satisfies outer boundary condition $T_{\nu}=0$ at the disc outer radius $r_{d}$. The unit of radius on the plot is $r_{d}$ in the case. The solid line is for a case that models the effects of the tidal field. The tidal field acts to sharply truncate the disc outside of the truncation radius $r_{\text {trunc }}$. The density distribution in this case satisfies satisfies boundary condition (70) and the unit of radius in the plot is $r_{\text {trunc }}$. The region $r>r_{\text {trunc }}$, where the density drops to zero, is not shown. There are additional corrections to this profile due to the effects of gas pressure in the outer parts of the disc.

Canup \& Ward (2002) have also considered the flow outside some radius where mass is injected, but did not include the effects of the tidal field. They have a normal accretion disc in the inner parts, as we have. But in the outer parts of the disc in their model, the material flows outwards and is removed from the disc at a rate $0.8 \dot{M}_{\mathrm{inj}} \sqrt{r_{\mathrm{inj}} / r_{\mathrm{d}}}$ where $r_{\mathrm{d}}$ is the outer edge of disc. For their standard model with $r_{\mathrm{inj}}=30 r_{\mathrm{J}}$ and $r_{\mathrm{d}}=150 r_{\mathrm{J}}$ (for Jupiter radius $r_{\mathrm{J}}$ ), we find the mass loss rate is $0.36 \dot{M}_{\mathrm{inj}}$. If we take the disc radius to be the orbit crossing radius, the mass loss rate is about $0.25 \dot{M}_{\text {inj. }}$ They suggest that the removal of matter at the outer edge of the disc occurs through solar torques or collisions with highly shocked regions. However, in our model the tidal torque dominates the viscous torques in the outermost regions and prevents the outward flow of matter. Hence, in our picture of a steady state, the rate that mass is accreted on to the central planet is the same rate that it is injected into the disc. In addition, there is a difference in the density profiles between the models outside the mass injection radius. In that region, Canup \& Ward (2002) determined a gradual tapering that varies in radius as $\sqrt{r_{\mathrm{d}} / r}-1$ for disk radius $r_{\mathrm{d}}$. In our model, the density falls off more slowly in radius in the main body of the disc and is abruptly terminated near the orbit crossing radius. The difference in the density structures can be traced to outer boundary conditions. Canup \& Ward (2002) employed a zero viscous stress outer boundary condition, $T_{\nu}=0$. In our model, the effects of the tidal field can be approximately represented by an outer boundary condition at the tidal truncation radius (orbit crossing radius) that is given by

$$
T_{\nu}=-\dot{M}_{\mathrm{inj}} r_{\mathrm{inj}}^{2} \Omega_{\mathrm{inj}},
$$

as follows from the second relation of equation (66) with $C_{2}=0$. Fig. 5 compares the density distributions resulting from the two boundary conditions. The density distribution we obtain approaches the Canup \& Ward (2002) distribution in the limit that the injection radius goes to zero. There are further modifications to the density profile due to pressure effects that are described in Section 8

From equation (69), the width of the disc outer edge (ignoring pressure effects) is estimated as

$$
w=1.5 \nu \Omega r\left|\frac{d T_{\mathrm{gr}}}{d M}\right|^{-1},
$$

where the right-hand side is evaluated near the outer edge. If the disc flow is smooth and there are no resonances, the phase lag that produces a tidal torque due to the turbulent viscosity is estimated as $\delta \theta \sim \nu /\left(r^{2} \Omega\right)$, the inverse Reynolds number of the flow. The resulting torque per unit disc mass is then estimated as $\left|d T_{\mathrm{gr}} / d M\right| \sim \delta \theta\left|\Phi_{2}\right|^{2} /\left(r^{2} \Omega^{2}\right)$, where $\Phi_{2}$ is the $m=2$ component of the tidal potential. Using equation (71) and assuming $\left|\Phi_{2}\right| \ll r^{2} \Omega^{2}$, it follows that $w \gg r$. Therefore, this torque is not capable of truncating the disc. Instead, the much stronger tidal torque, due to orbit crossings and certain resonance effects discussed in Section 8 , truncates the disc in a small region of space. Torques from the region well inside of where orbit crossings occur are then too weak to truncate the disc.

The analytic solutions can also be considered to apply when the matter being injected into the disc has a nonKeplerian velocity, that is $\Omega_{\mathrm{inj}}$ at $r_{\mathrm{inj}}$ is nonKeplerian. The gas in such a case adds angular momentum per unit mass to the system at a rate that differs from the Keplerian rate. In reality, such gas would undergo a strong Kelvin Helzholtz instability with the disc. Since the gas being added to the disc has a much lower density than the disc, we would expect this gas to be rapidly entrained by the disc that remains in nearly Keplerian rotation. An example of such a situation occurs when coplanar inflowing gas meets the circumplanetary disc at the disc outer edge. We expect the inflowing gas in this case to be subKeplerian. In such a case, the decretion disc or mass reservoir region, as depicted in Fig. 4 is very small. Instead, nearly entire disc behaves an accretion disc. The subKeperian injected gas can be considered to impart a negative torque on an otherwise Keplerian disc, in addition to the tidal torque. On the other hand, if much of the inflowing gas flows over the disc before becoming entrained by the disc, then the injection radius will be smaller than the disc outer radius, as in the cases plotted in Fig. 3 . 


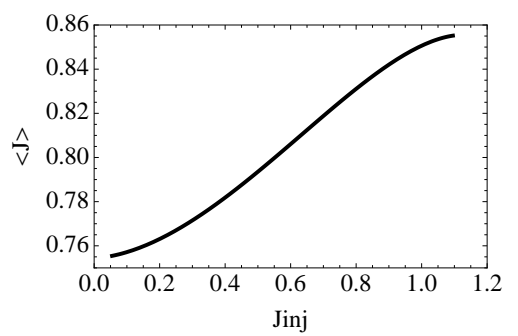

Figure 6. Average specific angular momentum (angular momentum per unit mass) $\langle J\rangle$ in a circumplanetary disc (total disc angular momentum divided by disc mass), based on the steady state model of Section 6.5, plotted as a function of the specific angular momentum of the accreting gas $J_{\mathrm{inj}}$. The quantities on the horizontal and vertical axes are normalized by $r_{\mathrm{H}}^{2} \Omega_{\mathrm{p}}$. The range of the horizontal axis extends to $J_{\text {inj }}$ at the disc outer edge, $r=0.41 r_{\mathrm{H}}$. The results show that the average specific disc angular momentum is nearly independent of the specific angular momentum of the accreting gas, even for small values. This model assumes that the gas flows over the disc and is entrained vertically at the local Keplerian speed for the mass injection radius. If planar subKeperian gas is added at the disc outer edge, we expect the variations of $\langle J\rangle$ to be even smaller, since the gas distribution within the main body of the disk behaves as a simple accretion disc.

Beyond the details of the flow properties in particular models, the results suggest that the disc structure is generally largely independent of the angular momentum per unit mass of the inflowing gas, as seen in Figs. 3 and 6 The disc density distribution is mildly influenced by this quantity. The radial derivative of the surface density undergoes order unity changes at $r_{\text {inj. }}$. In the model considered this section, the logarithmic surface density gradient changes from $-1 / 2$ to -1 across $r_{\text {inj. }}$ Also, for a fixed mass injection rate, the density in the outer parts of the disc scales as $\sqrt{r_{\text {inj }} / r_{\text {trunc }}}$. But such variations do not lead to strong changes in disc structure by typically plausible values of $r_{\mathrm{inj}}>0.05 r_{\mathrm{H}}$. This is particularly true if the injection radius is near the disc outer edge. Instead, the disc structure interior to the disc tidal truncation region is dominated by the effects of disc turbulent viscosity.

\section{DISC MODEL WITH PRESSURE}

To include the effects of gas pressure, we modeled the disc using a two-dimensional SPH code with $10^{5}$ particles. The SPH parameter $\alpha_{\mathrm{SPH}}$ was set to unity in these simulations, while $\beta_{\mathrm{SPH}}$ was set to zero. The disc sound speed was crudely modeled as a constant equal to $0.3 \mu^{1 / 3} a \Omega_{\mathrm{p}}=0.43 \Omega_{\mathrm{p}} r_{\mathrm{H}}$ throughout. The disc aspect ratio was $H / r \simeq 0.16$ at $R=0.4 R_{\mathrm{H}}$ (in dimensionless units of these equations $R_{\mathrm{H}}=3^{-1 / 3}$ ), where free particle orbit crossings occur. The fluid equations were taken in the Hill approximation, following the force equation (30). The particles were initially distributed so that surface density was initially $\Sigma(r, \theta) \propto 1 / R$ in an annulus $R_{\text {in }}<R<R_{\text {out }}$. We chose $R_{\text {in }}=0.07 R_{\mathrm{H}}$ and have two models, one with $R_{\text {out }}=0.3 R_{\mathrm{H}}$ and the second with $R_{\text {out }}=0.6 R_{\mathrm{H}}$ which we plot in Fig. 7. The initial velocities were taken to be circular rotation having angular speed $\Omega-\Omega_{\mathrm{p}}$ in the corotating frame, with $\Omega$ given by equation (24). Particles are removed from the simulation if they reach the inner boundary at $R=0.07 R_{\mathrm{H}}$ or the outer boundary located at $R=0.86 R_{\mathrm{H}}$. For each particle removed, a particle is injected at a random angle and random radius between $R=0.22 R_{\mathrm{H}}$ and $R=0.36 R_{\mathrm{H}}$. Therefore, the number of particles is fixed at $10^{5}$ at all times.

Fig. 8 plots the angular momentum evolution as a function of time for the two initial disc sizes. The smaller disc starts with much less angular momentum than the larger one. But after only about 3 planetary orbits both discs have similar values of angular momentum and approach a steady state. In Fig. 9 we plot the particles at a time of 6 planet orbital periods. We see that particle distributions look very similar and the disc has reached a near steady state. The viscous timescale of the disc can be estimated as $\sim r^{2} / \nu$. In SPH, we have that $\alpha \simeq 0.1 \alpha_{\mathrm{SPH}}=0.1$ (e.g., Artymowicz \& Lubow 1994). The value of the kinematic viscosity $\nu$ is estimated as $0.1 \alpha_{\mathrm{SPH}}(H / r)^{2} r^{2} \Omega$ and the viscous timescale evaluates to about 8 orbits at $R \simeq 0.3 R_{\mathrm{H}}$. The simulated disc has therefore settled to a near equilibrium state on a timescale that is of order the estimated viscous timescale. This kinematic viscosity is a factor of ten or more larger than what is typically taken taken in circumplanetary disc simulations (e.g., D'Angelo et al. 2002; Ayliffe \& Bate 2009). Consequently, in those simulations, the timescales to reach a steady state are longer than the case here by similar factor. (In addition, previous simulations have generally not started with a circumplanetary disc. Some time is required for its formation from inflowing gas.) For the simulations in Machida (2009), the timescale would be determined by the inherent viscosity in the code due to the finite differencing, since no viscous terms were included.

The phasing of the arms determines the sign of the gravitational torque in the different regions of space as shown in Fig. 10] The left plot shows the cumulative gravitational torque as a function of radius in a steady state disc after 6 planetary orbits. On the right is a plot of the shape of the spiral in the disc. The sign of the gravitational torque is equal to sign of $-x y$ and so is negative in the first and third quadrants the torque and positive in the second and fourth. As seen in the left plot, the gravitational torque is negative in the outermost region of the disc where it is strongest. The spiral there lies in the 

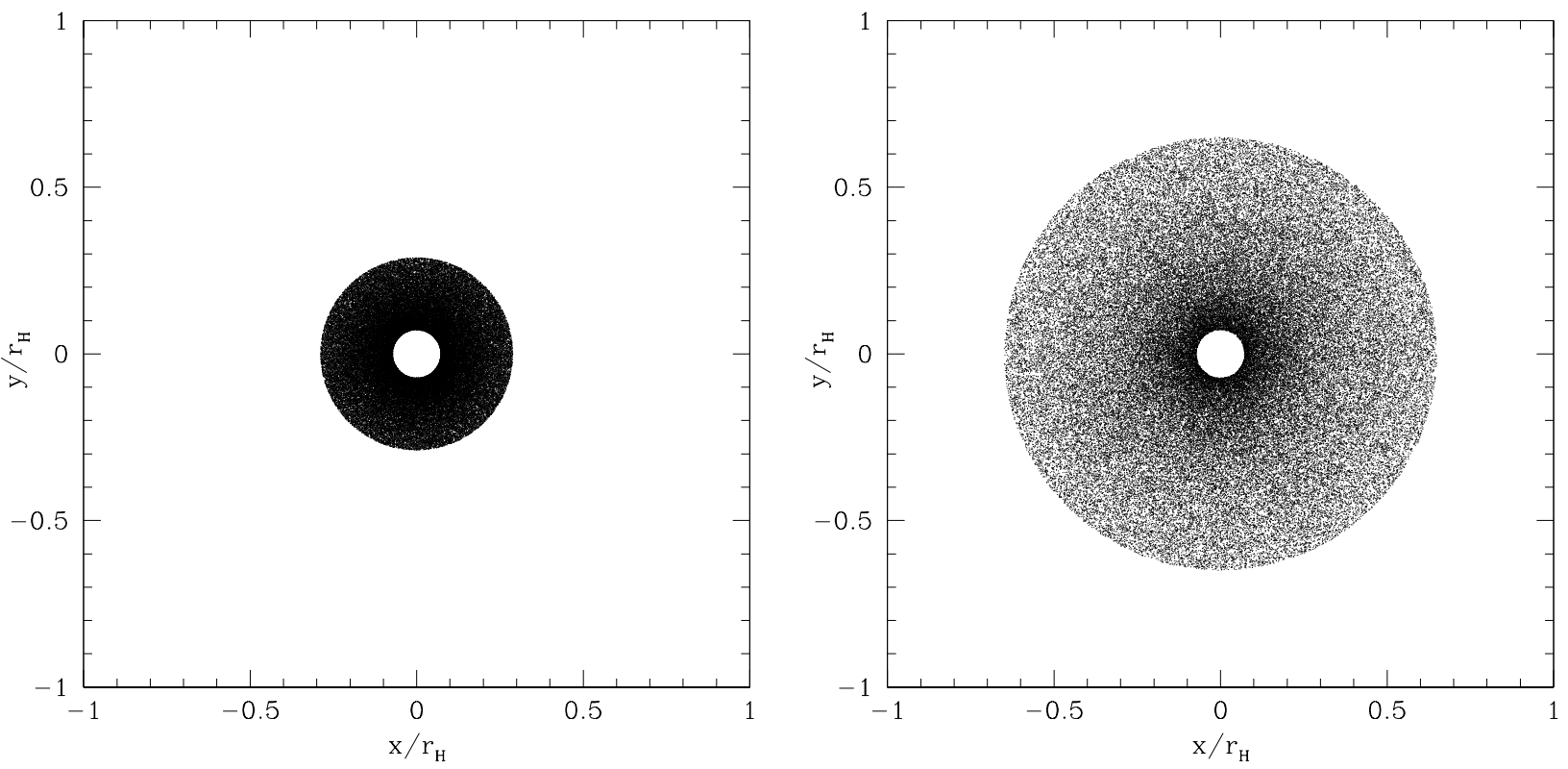

Figure 7. The initial configuration for the two SPH runs. Left: the particles are randomly distributed in radius of $0.07 r_{\mathrm{H}}<r<0.3 r_{\mathrm{H}}$. Right: The particles are randomly distributed randomly in angle and randomly in radius within and annulus defined by $0.07 r_{\mathrm{H}}<r<$ $0.65 r_{\mathrm{H}}$.
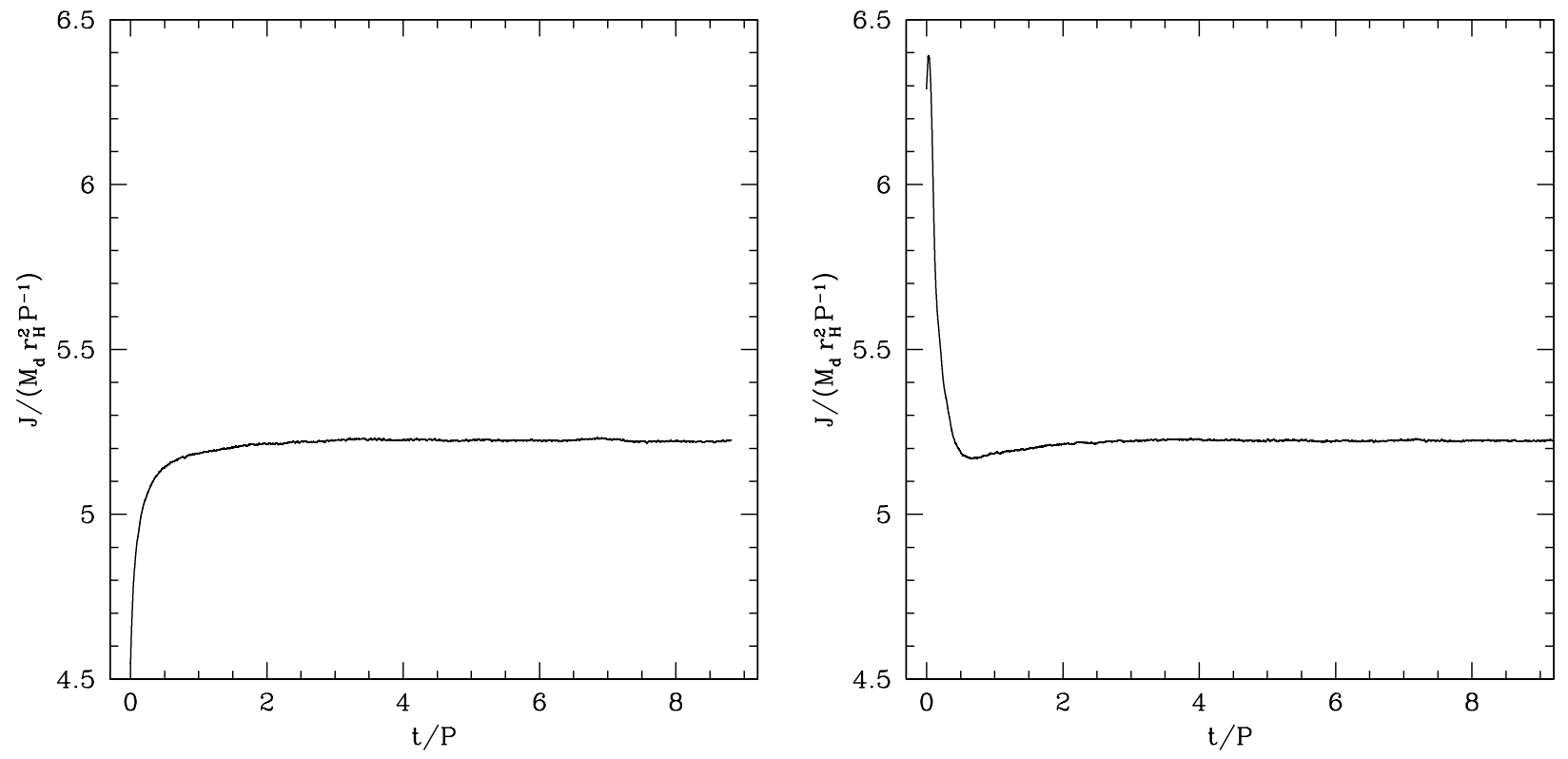

Figure 8. The angular momentum evolution in time for the initially small disc (left) and the large disc (right), plotted in Fig. 7 The units of angular momentum are $M_{\mathrm{d}} r_{\mathrm{H}}^{2} P^{-1}$, for a disc of mass $M_{\mathrm{d}}$. The unit of time is the planet orbit period, $P$.

first and third quadrants, as seen in Fig. 9 in order the provide a net negative gravitational torque that removes angular momentum from the disc as gas accretes.

The left plot in Fig. 10also shows the cumulative disc mass distribution. More than $90 \%$ of the disc mass is located inside a radius of $0.41 r_{\mathrm{H}}$, where orbits crossings of free particles occur. The local gravitational torque is negative for $r>0.35 r_{\mathrm{H}}$. On the other hand, the cumulative gravitational torque on the disc inside the orbit crossing radius is actually positive. This does not mean that the gas gains angular momentum in this region. The viscous torques compensate by transporting the angular momentum out of this region so that the overall torque is negative. The negative cumulative gravitational disc torque is achieved somewhat outside the orbit crossing radius. A small amount of disc mass, less than $10 \%$, that is located outside that 

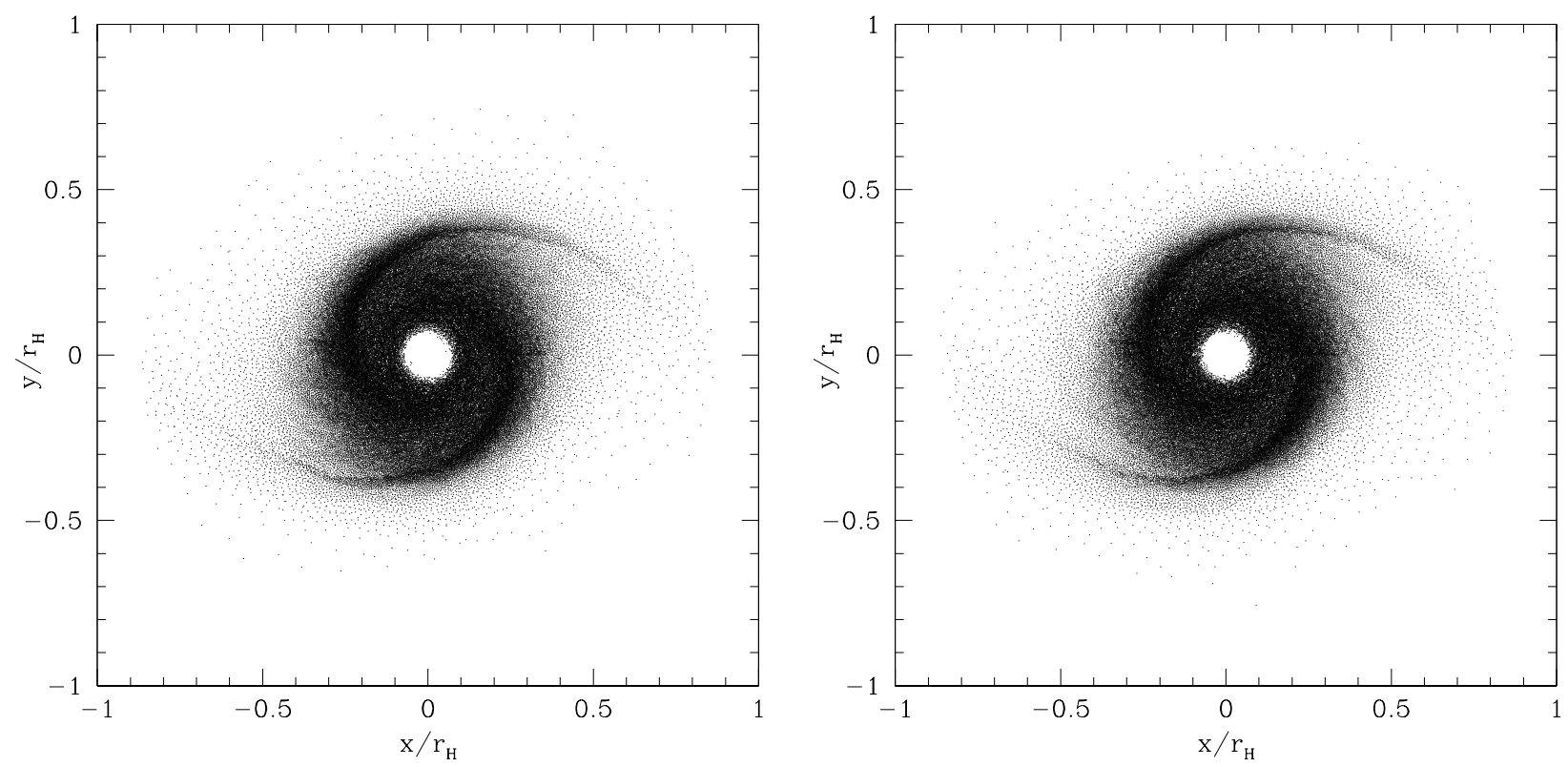

Figure 9. The particle distribution in space for the two SPH simulations corresponding to those with the initial configuration in Fig. 7 at a time of 6 planetary orbital periods.
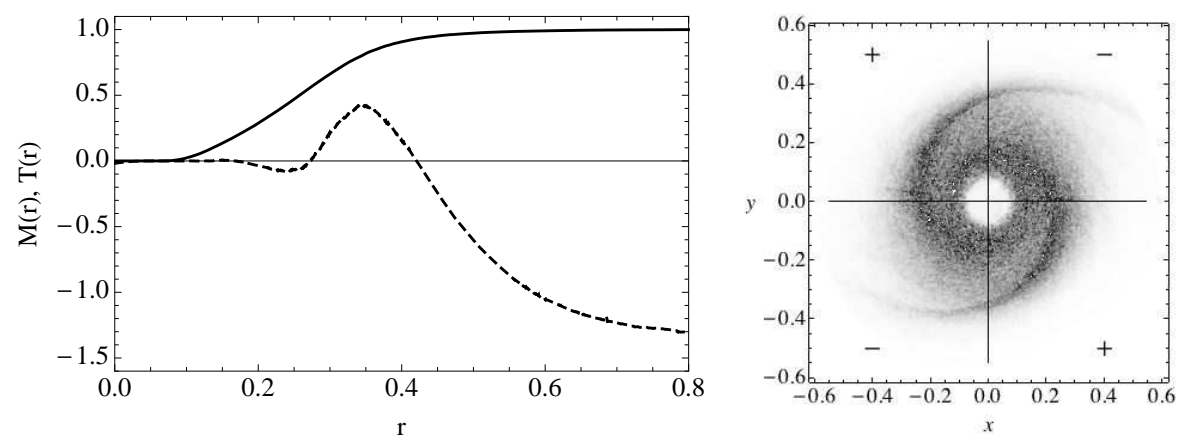

Figure 10. Left: Cumulative disc mass (solid) in units of total disc mass $M_{\mathrm{d}}$ and cumulative gravitational torque (dashed) on the disc in units of $0.01 M_{\mathrm{d}} r_{\mathrm{H}}^{2} \Omega_{\mathrm{p}}^{2}$ starting at the disc center as a function of radius in units of $r_{\mathrm{H}}$ at a time of 6 planetary orbits. Right: Plot of the particle positions in units of $r_{\mathrm{H}}$ with the point size weighted by density, in order to enhance the visibility of the spiral wave in the main body of the disc. The sign of the gravitational torque in each quadrant is also shown. At small radii, $r \simeq 0.2$, the spiral lies in the first and third quadrants where the gravitational torque on the disc is negative, and the cumulative gravitational torque begins as negative in the left panel. At $r \simeq 0.3$, the spiral lies in the second and fourth quadrants where the gravitational torque on the disc is positive, and the cumulative gravitational torque turns positive. The outer parts of the spiral lie in the first and third quadrants and provide a negative gravitational torque contribution that causes the total torque on the disc to be negative.

radius provides the net negative torque on the disc. In that region of space, $r \gtrsim 0.41 r_{\mathrm{H}}$, the spiral arms are very prominent in Figure 9 and gas response is highly nonlinear. A similar spiral structure was found in the case of a warm disc with $H / r=0.1$ within a binary star system by Savonije et al. (1994) that we have argued should be similar to the circumplanetary disc case (see Section 4).

The SPH code is less accurate in these outer low density regions because the interparticle pressure force calculation is more approximate. Also, we have simplified the inflow on to the disc to be occurring within an annulus in the disc. But, we expect that the general properties of gravitational torque to generally hold. That is, the gravitational torque involves a relatively small amount of gas in the outer parts of disc.

\section{DISCUSSION AND CONCLUSIONS}

We have analysed the dynamics of a circumplanetary disc as an accretion disc subject to the tidal forces of the central star. We applied several techniques: ballistic particle orbits, one-dimensional simulations and analytic models, and two-dimensional 


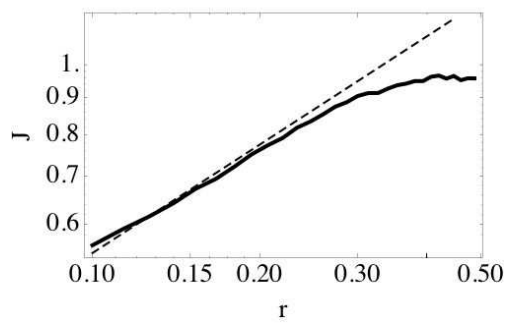

Figure 11. Angular momentum per unit disc mass, $J$, in units of $\Omega_{\mathrm{p}} r_{\mathrm{H}}^{2}$ plotted against distance from the planet in units of $r_{\mathrm{H}}$ on a $\log -\log$ scale. The solid line is obtained from the SPH simulations described in Section 7 at a time of 6 orbits, while the dashed line is the Keplerian case. The solid curve is based on binning in 30 radial bins, in order to smooth fluctuations. For $r \simeq 0.3$, inside of where orbits cross $(r \simeq 0.4), J$ in the disc departs from the Keplerian case due to the effects of gas pressure as the disc tapers. The outer disc velocity is somewhat subKeplerian because the pressure forces act radially outward.

SPH simulations. We have shown that the gas dynamical equations for a circumplanetary disc can be rescaled to a form that is similar to those previously analysed for a disc in a binary star system with mass ratio of unity, but with a modified (Hill) potential. The size of a circumplanetary disc is determined by the requirement that tidal torques from the central star remove angular momentum at a rate required by steady inflow on to the planet. In the crudest approximation that is accurate for a cold disc, disc streamlines consist of a set of stable, nested periodic ballistic particle orbits (see Fig. 1). For a cold disk, the disc radius is determined to be the location of orbit intersections or instability that occurs at $0.41 r_{\mathrm{H}}$, for Hill radius $r_{\mathrm{H}}$. This radius is close to the effective disc radius (inside of which $\sim 90 \%$ of the mass is located) found in the $\mathrm{SPH}$ simulations for a warm disc (see Figs. 9 and 10). In principle, the observational determination of the size of this disc then provides a constraint on the planet mass.

In some previous disc models, the angular momentum of the inflowing gas was thought to control the disc size. According to these models, the compactness of the giant planet satellite systems, within a region of radius much less than $r_{\mathrm{H}}$, is a consequence of a low angular momentum inflow that penetrates well inside the Hill sphere and deposits presatellite material there (e.g. Lissauer 1995; Canup \& Ward 2002; Mosqueira \& Estrada 2003; Ward \& Canup 2010). We suggest a different physical model for the processes that control the circumplanetary disc size and structure. We find that although the angular momentum per unit disc mass (specific angular momentum) of the inflowing gas plays a role in determining whether a circumplanetary disc can form, it has a minor influence on the disc structure. A steady state accretion disc does not achieve as compact a form as desired by these previous models. These results imply that the common scale of the disc seen in the simulations by Avliffe \& Bate (2009) and D'Angelo et al. (2002) is not due to the specific angular momentum of the inflow, but instead to the tidal truncation effects. The angular momentum within the disc is redistributed by viscous torques. The disc structure inside the truncation radius is mainly determined by the properties of the disc turbulent viscosity, rather than the specific angular momentum of the inflowing gas (see uppermost curves in Fig. 3). The average specific angular momentum in the disc is nearly independent of the specific angular momentum of the accreting gas (see Fig. 6). Tidal effects have a large-scale influence on the disc density distribution, as discussed in Section 6.5.

We have concentrated on the case that the planet opens a gap in the disc, as is expected for a Jupiter mass planet. Some previous studies suggested that a small disc of size $\sim r_{\mathrm{H}} / 48$ could result prior to and during early stages of gap opening (e.g. Estrada et al. 2008). Our results suggest that a turbulent accretion disc would be truncated at the orbit crossing radius, even if the inflowing gas has low specific angular momentum. There are are few qualifications in this statement. The simulations of Ayliffe \& Bate (2009) found that circumplanetary discs do not exist prior to gap opening (planet masses less than 100 Earth masses). If low angular momentum gas is accreted in early stages of gap opening, then pressure forces from within the Hill sphere could prevent a disc from extending out to the orbit crossing radius, if the planet's Bondi radius is sufficiently smaller than its Hill radius. Under such conditions, we would expect the disc to expand to the Bondi radius by viscous torques, since tidal torques would not be adequate to truncate the disc inside the Bondi radius. The disc would then need to lose its angular momentum to the surrounding gas in the circumstellar disc. Whether such a configuration is possible is unclear. In addition, gap opening or planet envelope contraction (below its Hill sphere) might not begin when the Bondi radius is smaller than the Hill radius and so a disc may not form under such conditions.

During the $\mathrm{T}$ Tauri accretion phase, circumplanetary disc aspect ratios are expected to be large, $H / r \sim 0.3$, as a consequence of the weak gravitational forces due to the planet and relatively high temperatures (see equation (4)). There are some implications of these high disc aspect ratios. One is that the disc edge tapering is more gradual than in the thin disc case. The tapering must be on a scale $\gtrsim H$ in order to avoid instabilities that occur for sharper edges (e.g. Yang \& Menou 2010). The tapering can then have an influence on a substantial portion of the circumplanetary disc. Avliffe \& Bate (2009) estimated the disc outer radius based on where the angular momentum per unit disc mass departs from Keplerian and begins to decline in radius. They obtained a disc outer radius of $\simeq 0.35 r_{\mathrm{H}}$ (see the upper right panel of their Fig. 2). This radius 
is somewhat inside the orbit crossing radius of $\simeq 0.41 r_{\mathrm{H}}$. The effect of the disc tapering on the distribution of disc angular momentum in our SPH simulations can be seen in Fig. 11] It leads to subKeplerian velocities inside the orbit crossing radius, similar to the results of Ayliffe \& Bate (2009). The results of these two studies can be further reconciled by considering the differences in the models. In their simulations, the disc is hotter than in our simulations. Consequently the pressure forces and their effects on departures from Keplerian rotation in the outer parts of the disc should be stronger than in Fig. 11 as is consistent with their results. Also the work of Ayliffe \& Bate (2009) includes the inflowing gas from larger radii that is omitted in our simulations. This inflowing subKeplerian gas and its interaction with the disc outer edge as it becomes entrained could further modify the results plotted in Fig. 11 It is also possible that the disc radius is affected by the location of wave damping, as discussed below. Such effects should be explored further.

Another implication of the high values of $H / r$ is that a small fraction of the disc mass can reside at larger disc radii than the orbit-crossing radius and so be subject to the effects of resonances that lie outside this radius, as discussed in Section 5 (see Fig. 10). In addition, off-resonant tidal forcing of the outer parts of the disc that lie inside the orbit crossing radius can play an important role. The resonance width depends on $(H / r)^{2 / 3}$ and is not small. The substantial width can allow the resonant region to overlap with the denser parts of disc. As a result of these two effects, two-armed spiral waves can be launched in such discs, even though exact resonances do not lie within the main body of the discs. We have found evidence of such waves in our SPH simulations (see Fig. 9). For these waves to play a role in extracting angular momentum from the disc, they must damp in order to introduce irreversibility. Otherwise, a standing wave is produced that results in little or no torque on the disc. Some damping can be produced by the disc turbulent viscosity in the fractional amount that is roughly the ratio of the viscous wave damping rate to the wave group propagation rate, about $\alpha(r / H)$. This ratio can be quite small for $\alpha \ll H / r$ and the torque is reduced to a similarly small fraction of its potentially maximum value. For a thin disc, this damping could occur as launched waves propagate, steepen and shock, since their wavelengths are short compared to the disc radius. But for warm circumplanetary discs, this process is less important because the wavelengths are not short compared to the disc radius. In the SPH simulations of Section 7, the damping seems to occur from the strongly nonlinear forcing in the outer disc, similar to the case of mildly warm discs $(H / r=0.1)$ previously investigated for binary star systems (Savonije et al. 1994). The SPH simulations suggest that the negative torque is produced in the outer parts of the disc, somewhat inside and beyond the radius where nested particle orbits cross or become unstable. This torque involves a relatively small amount of disc mass (see Fig. 10). However, the discs in our SPH simulations were two-dimensional and not as warm as could occur, where $H / r \sim 0.3$. The wave damping issue should be explored in future work.

The accretion disc model does not provide an obvious explanation for the locations of the regular satellites of Jupiter and Saturn that occur within $0.06 r_{\mathrm{H}}$ of the planet. As discussed above, the disc structure is insensitive to the angular momentum of the inflowing gas and satellites lie well inside the tidal truncation radius of the disc. This conclusion is consistent with the results of recent three-dimensional simulations of Estrada et al. (2008) and Avliffe \& Bate (2009). We have considered circumplanetary discs with a smoothly varying turbulent viscosity with radius. But such discs could also harbor dead zones, as discussed in Section 2 Such situations could result in rapid disc density variations at their boundaries. The existence of satellites requires survival against the effects of migration. Such density variations could affect satellite migration and possibly trapping satellites, since migration rates depend on density gradients (e.g., Matsumura, Pudritz \& Thommes 2007). But, if the inner dead zone boundary is due to thermal ionisation at a temperature of $10^{3} \mathrm{~K}$, then the disc temperatures there would be too hot for the survival of an icy satellite. Of course, other sources of ionisation could change the locations of dead zone edges. In addition, the nonlinear feedback from waves in a low viscosity disc could slow migration (Ward 1997; Rafikov 2003; Li et al. 2009). However, this slowing is less effective for the warmer circumplanetary discs. If the satellite formation occurs after partial disc depletion, as suggested by Canup \& Ward (2002), then the ionisation through the vertical extent of the disc becomes easier and the dead zones are less likely to occur at that stage.

Circumplanetary discs are not very bright as we see in equation (8). Time dependent accretion and outbursts could occur

in dead zones, as has been suggested to explain FU Orionis outbursts in young stellar systems Armitage. Livio \& Pringle 2001). During a circumplanetary disc outburst, the ratio of the circumplanetary to circumstellar luminosities could be much higher.

\section{ACKNOWLEDGMENTS}

We thank Matthew Bate, Gennaro D'Angelo, and Jim Pringle for helpful discussions. RGM thanks the Space Telescope Science Institute for a Giacconi Fellowship. SHL acknowledges support from NASA grant NNX07AI72G.

\section{REFERENCES}

Armitage P. J., Livio M., Pringle J. E., 2001, MNRAS, 324, 705

Artymowicz P., Lubow S. H., 1994, ApJ, 421, 651 
Artymowicz P., Lubow S. H., 1996, ApJ, 467, L77

Ayliffe B. A., Bate M. R., 2009, MNRAS, 397, 657

Barr A. C., Canup R. M., 2008, Icarus, 198, 163

Bate M. R., Lubow S. H., Ogilvie G. I., Miller K. A., 2003, MNRAS, 341, 213

Börner G., Meyer F., Schmidt H. U., Thomas H. C., 1973, Mitt. der. astr. Ges., 32, 237

Bryden G., Chen X., Lin D. N. C., Nelson R. P., Papaloizou C. B., 1999, ApJ, 514, 344

Ćuk M., Burns J. A., 2004, Icarus, 167, 369

Canup R. M., Ward W. R., 2002, ApJ, 124, 3404

D'Angelo G., Henning T., Kley W., 2002, A\&A, 385, 647

Estrada P. R., Mosqueira I. L., Jack J., D’Angelo G., Cruikshank D. P., 2008, arXiv0809.1418E

Frank J., King A., Raine D., 2002, Accretion Power in Astrophysics, Cambridge University Press, Cambridge

Goldreich P., Tremaine S., 1979, ApJ, 233, 857

Gomes R., Levison H. F., Tsiganis K., Morbidelli A., 2005, Nat, 435, 466

Grav. T., Holman M. J., Gladman B. J, Aksnes K., 2003, Icarus, 166, 33

Henon, M. 1965, Ann. d'Ap, 28, 995

Hubickyj O., Bodenheimer P., Lissauer J. J., 2005, Icarus, 179, 415

Kley W., 1999, MNRAS, 303, 696

Li H., Lubow S. H., Li S., Lin D. N. C., 2009, ApJ, 690, 52

Lin D. N. C., Pringle J. E., 1974, Proc. IAU Symp., 73, 237, eds Eggleton P. P. et al., Reidel, Dordrecht

Lin D. N. C., Papaloizou J.,1986, ApJ, 309, 846

Lissauer J. J., 1995, Icarus, 114, 217

Lissauer J. J., Hubickyj O., D'Angelo G., Bodenheimer P., 2009, Icarus, 199, 338

Lubow S. H., 1981, ApJ, 245, 274

Lubow S. H., Seibert M., Artymowicz P., 1999, ApJ, 526, 1001

Lubow S. H., D’Angelo G., 2006, ApJ, 641, 526

Lunine, J. I., \& Stevenson, D. J. 1982, Icarus, 52, 14

Machida, 2009, MNRAS, 392, 514

Martin R. G., Lubow S. H., Pringle J. E., Wyatt M. C., 2007, MNRAS, 378, 1589

Matsumura S., Pudritz R. E., Thommes E. W., 2007, ApJ, 660, 1609

Mizuno H., 1980, Prog. Theor. Phys., 64, 544

Mosqueira I., Estrada P. R., 2003, Icarus, 163, 198

Paczynski B., 1977, ApJ, 216, 822

Papaloizou J., Pringle J. E., 1977, MNRAS, 181, 441

Papaloizou, J. C. B., Nelson, R. P. 2005, A\&A, 433, 247

Pollack J. B., Burns J. A., Tauber M. E., 1979, Icarus, 7, 587

Pollack J. B., Hubickyj O., Bodenheimer P., Lissauer J. J., Podolak M., Greenzweig Y., 1996, Icarus, 124,62

Pringle J. E., 1981, ARA\&A, 19, 137

Quillen A. C., Trilling D. E., 1998, ApJ, 508, 707

Rafikov R. R., 2003, AJ, 126, 2529

Savonije G. J., Papaloizou, J. C. B., Lin D. N. C., 1994, MNRAS, 268, 13

Shakura N. I., Sunyaev R. A., 1973, A\&A, 29, 179

Terquem C. E. J. M. L. J., 2008, ApJ, 689, 532

Ward W. R., 1997, Icarus, 126, 261

Ward W. R., Canup, R.M. 2010, AJ, 140, 1168

Yang, C.-C., Menou K., 2010, MNRAS, 402, 2436 